\title{
One-Dimensional Edge Contacts to a Monolayer Semiconductor
}

\section{Journal Article}

\section{Author(s):}

Jain, Achint (D); Szabó, Áron; Parzefall, Markus (D); Bonvin, Eric (D); Taniguchi, Takashi; Watanabe, Kenji; Bharadwaj, Palash; Luisier, Mathieu; Novotny, Lukas (iD)

Publication date:

2019-10-09

Permanent link:

https://doi.org/10.3929/ethz-b-000372768

Rights / license:

In Copyright - Non-Commercial Use Permitted

Originally published in:

Nano Letters 19(10), https://doi.org/10.1021/acs.nanolett.9b02166

Funding acknowledgement:

165841 - Nonlinear optics with hybrid plasmonic-TMDC materials (SNF) 


\title{
One-dimensional edge contacts to a monolayer semiconductor
}

\author{
Achint Jain, ${ }^{1, *}$ Áron Szabó,${ }^{2}$ Markus Parzefall, ${ }^{1}$ Eric Bonvin, ${ }^{1}$ Takashi Taniguchi, ${ }^{3}$ \\ Kenji Watanabe, ${ }^{3}$ Palash Bharadwaj, ${ }^{4}$ Mathieu Luisier, ${ }^{2}$ and Lukas Novotny ${ }^{1, \dagger}$ \\ ${ }^{1}$ Photonics Laboratory, ETH Zürich, 8093 Zürich, Switzerland \\ ${ }^{2}$ Integrated Systems Laboratory, ETH Zürich, 8092 Zürich, Switzerland \\ ${ }^{3}$ National Institute for Material Science, 1-1 Namiki, Tsukuba 305-0044, Japan \\ ${ }^{4}$ Department of Electrical and Computer Engineering, Rice University, Houston, TX 77005, USA
}

(Dated: October 17, 2019)

\begin{abstract}
Integration of electrical contacts into van der Waals ( $\mathrm{vdW}$ ) heterostructures is critical for realizing electronic and optoelectronic functionalities. However, to date no scalable methodology for gaining electrical access to buried monolayer two-dimensional (2D) semiconductors exists. Here we report viable edge contact formation to hexagonal boron nitride $(\mathrm{hBN})$ encapsulated monolayer $\mathrm{MoS}_{2}$. By combining reactive ion etching, in-situ $\mathrm{Ar}^{+}$sputtering and annealing, we achieve a relatively low edge contact resistance, high mobility (up to $\sim 30 \mathrm{~cm}^{2} \mathrm{~V}^{-1} \mathrm{~s}^{-1}$ ) and high on-current density ( $>50 \mu \mathrm{A} / \mu \mathrm{m}$ at $\left.V_{\mathrm{DS}}=3 \mathrm{~V}\right)$, comparable to top contacts. Furthermore, the atomically smooth $\mathrm{hBN}$ environment also preserves the intrinsic $\mathrm{MoS}_{2}$ channel quality during fabrication, leading to a steep subthreshold swing of $116 \mathrm{mV} / \mathrm{dec}$ with a negligible hysteresis. Hence, edge contacts are highly promising for large-scale practical implementation of encapsulated heterostructure devices, especially those involving air sensitive materials, and can be arbitrarily narrow, which opens the door to further shrinkage of 2D device footprint.
\end{abstract}

Keywords: 2D materials, TMDCs, heterostructures, edge contacts, encapsulation

Two-dimensional electronic devices made from transition metal dichalcogenides (TMDCs) have gained prominence in recent years for next-generation integrated electronics ${ }^{1}$ and nanophotonics applications. In particular, $\mathrm{MoS}_{2}$ combined with other 2D materials into vdW heterostructures, appears as an attractive candidate for future transistor architectures ${ }^{2}$, atomically thin $\mathrm{p}-\mathrm{n}$ junctions and tunnel diodes ${ }^{3}$, memristors ${ }^{4}$, high-efficiency photodetectors ${ }^{5}$, light emitting diodes ${ }^{6}$ and novel valleytronic devices ${ }^{7}$. Such heterostructures are often assembled in a top-down manner by picking-up discrete 2D material layers with a top hBN flake and placing the resulting stack on a target substrate. Although the presence of a top hBN layer on one hand serves to encapsulate the constituent 2D materials in the heterostructure, at the same time however, it also hinders the fabrication of direct electrical contacts to the underlying layers. Despite vdW heterostructures having been extensively investigated, a practical route for making electrical contacts to them in a scalable manner is still lacking.

In TMDC heterostructures assembled without any encapsulation layer, further limitations arise when electrical contacts are made in a conventional top-contact geometry. In this scenario, the contact electrodes come in direct physical contact with a TMDC layer over a finite area. Since such a methodology inherently requires performing lithography on unprotected TMDC layers, it exposes them to foreign chemical species which are difficult to remove. Additionally, bare TMDC surfaces in air are susceptible to $\mathrm{O}_{2}$ and $\mathrm{H}_{2} \mathrm{O}$ adsorption ${ }^{8-10}$. Owing to their atomically thin nature however, mono- and few-layer TMDCs are quite sensitive to their immediate environment which includes both surface adsorbates from ambient exposure and processing residues. These act as unintentional dopants leading to a spatially inhomogeneous carrier density ${ }^{11}$, which causes device-to-device variations in threshold voltage $^{12,13}$ and Schottky barrier height ${ }^{14}$. Besides doping, surface contaminants also scatter ${ }^{15}$ and trap charge carriers, thereby resulting in reduced mobility, low on-current ${ }^{8-10}$, increased flicker noise $^{16,17}$, hysteresis ${ }^{18}$ and compromised optical properties ${ }^{19}$ Although measurements performed under high vacuum after in-situ annealing have made it possible to observe the intrinsic electrical transport properties of $\mathrm{MoS}_{2}{ }^{20,21}$, unencapsulated devices measured in air show a drastic reduction in carrier mobility, implying that even short-term air exposure is detrimental for monoand bi-layer $\mathrm{MoS}_{2}$ devices ${ }^{8,9}$.

Therefore, for enabling a viable usage of TMDCs in integrated electronics, better contact techniques are needed that allow for encapsulation before contact patterning, in order to preserve the intrinsic material quality and achieve superior performance. Moreover, encapsulation is also essential for long-term ambient stability since it is well-known that most TMDCs, including $\mathrm{MoS}_{2}, \mathrm{MoSe}_{2}$ and $\mathrm{WS}_{2}$ undergo gradual oxidation in air at room temperature ${ }^{22}$, which leads to further mobility degradation ${ }^{23}$, morphological changes ${ }^{24,25}$ and adversely affected photoluminescence ${ }^{26}$. In fact, some $2 \mathrm{D}$ materials like $\mathrm{MoTe}_{2}, \mathrm{HfSe}_{2}, \mathrm{ZrSe}_{2}, \mathrm{NbSe}_{2}$, black phosphorus and InSe, are so unstable in air that surface deterioration can be detected within a day ${ }^{25}$. This restricts their assembly to an inert atmosphere ${ }^{27}$ and encapsulation in hBN is commonly employed to limit air exposure ${ }^{23,27}$. With such materials, lithographic contact fabrication prior to encapsulation is not only difficult but also impractical.

In order to circumvent the issue of making electrical contacts to $\mathrm{hBN}-\mathrm{TMDC}-\mathrm{hBN}$ heterostructures, a common practice is to embed additional layers of graphene ${ }^{23,28,29}$ or metallic $\mathrm{NbSe}_{2}{ }^{30,31}$ within the stack to act as electrodes. Pre-patterning contact vias into the top hBN before pick-up ${ }^{32,33}$ or transfer of pre-patterned metal films onto TMDCs ${ }^{34}$ (or vice-versa) have also been reported. However, alignment and transfer of multiple contact layers severely increases the fabrication complexity, especially in multilayer heterostructures, and becomes difficult to scale-up for practical purposes. Moreover, in case of graphene, the contact resistance $\left(R_{\mathrm{C}}\right)$ sensitively depends on the twist angle between the graphene and TMDC layers which poses further alignment challenges 35 Even though large-area chemical vapor deposition (CVD) growth of lateral graphene-MoS 2 heterostructures has made progress in recent years $^{36-38}$, hard to control growth inhomogeneities ${ }^{38,39}$ as well as ripples and strain induced by lattice mismatch still exist along 2D-2D edge interfaces ${ }^{40}$, which could ultimately hinder fabrication of very short channel $(<100 \mathrm{~nm})$ devices. Another possibility is to fabricate tunneling contacts on encapsulated TMDCs ${ }^{41-44}$ However, such devices are restricted to very thin hBN (1-4L) or oxide $(\sim 2 \mathrm{~nm})^{45}$ encapsulation layers for optimum carrier injection. A more versatile approach is to etch through the top hBN layer in order to expose an edge of any buried 2D material of interest and form a one-dimensional (1D) 'edge contact' to it ${ }^{46,47}$. Although such a strategy has been highly successful for graphene ${ }^{46}$, similar attempts to make $1 \mathrm{D}$ edge contacts to monolayer (1L) $\mathrm{MoS}_{2} 48,49$ and few-layer $\mathrm{WSe}_{2}{ }^{50}$ were met with limited success until now.

Here we report reliable edge contact formation to $\mathrm{hBN}$ encapsulated $1 \mathrm{~L}-\mathrm{MoS}_{2}$. Our devices exhibit very low hysteresis together with a high mobility and steep subthreshold swing, highlighting the pristine interface quality achieved. By a systematic optimization of the fabrication process, we obtain a moderately low contact resistance and a high on-current $(>50 \mu \mathrm{A} / \mu \mathrm{m})$ with $\mathrm{Ti}-\mathrm{Au}$ edge contacts, despite a vanishingly small contact area. The contact performance remains unchanged even at low temperatures, making edge contacts promising for cryogenic experiments and applications. 
a

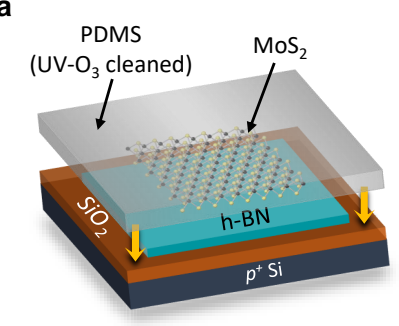

d

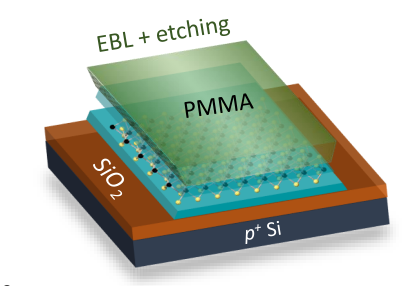

f

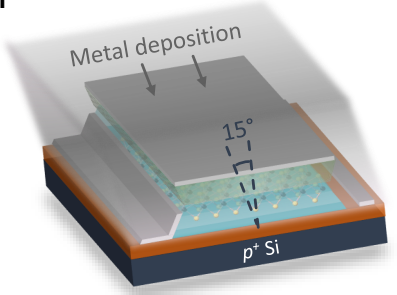

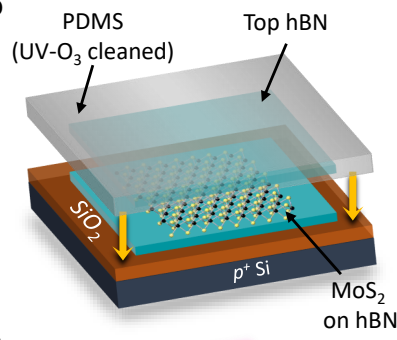

e

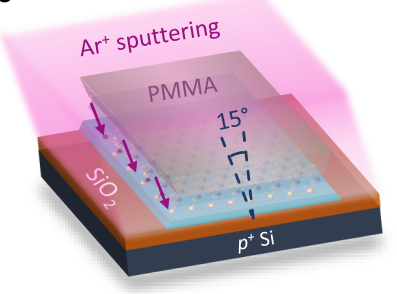

g

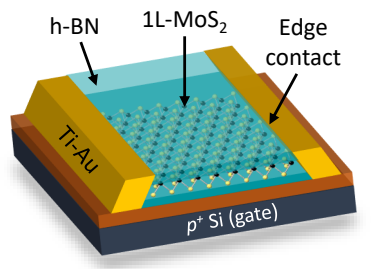

C

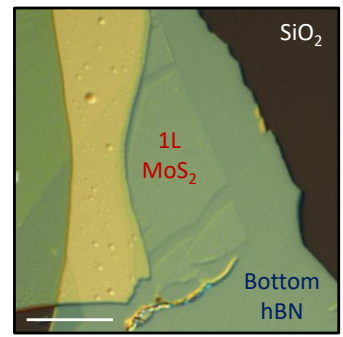

h

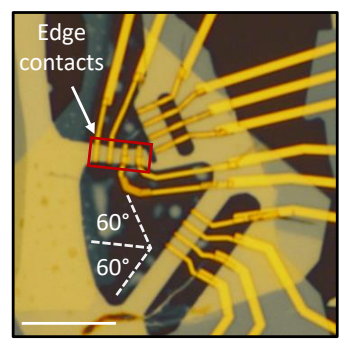

Figure 1. Fabrication of edge contacts. (a-b) 3D illustration of the heterostructure assembly. 1L-MoS 2 is exfoliated on PDMS and transferred onto an hBN layer. Subsequently, the $\mathrm{MoS}_{2}$ is fully encapsulated by stacking another hBN layer on top. UV-O 3 cleaning of the PDMS surface before exfoliating MoS 2 on it significantly reduces PDMS residues on $\mathrm{MoS}_{2}{ }^{51}$. (c) Differential interference contrast (DIC) optical image of a $1 \mathrm{~L}-\mathrm{MoS} 2$ flake transferred on hBN (24 nm thick) and vacuum annealed. (d-g) $3 \mathrm{D}$ illustration of edge contact fabrication. (d) The hBN-MoS 2 -hBN heterostructure is patterned by EBL and RIE to expose $\mathrm{MoS}_{2}$ edges. (e) Before metallization, in-situ $\mathrm{Ar}^{+}$sputtering is done at $+15^{\circ}$ and also $-15^{\circ}$, inside a UHV chamber. This creates clean $\mathrm{MoS} 2$ contact edges by removing $\mathrm{MoO}_{\mathrm{x}}$ and any adsorbed gas molecules. (f-g) $\mathrm{Ti}(5+5 \mathrm{~nm})$ is then immediately deposited followed by Au (40 + $\left.40 \mathrm{~nm}\right)$, both metals at $+15^{\circ}$ as well as $-15^{\circ}$, to form $1 \mathrm{D}$ edge contacts. (h) Optical image of the $\mathrm{MoS}_{2}$ sample shown in c, contacted via edge contacts after hBN encapsulation. In the devices outlined in red, the $\mathrm{MoS}_{2}$ contact edges were not sputtered with $\mathrm{Ar}^{+}$, to act as a control. Ar ${ }^{+}$treatment results in a lower contact resistance, as discussed later. The three sets of devices in $\mathbf{h}$ were aligned at $60^{\circ}$ with respect to each other, in order to exclude any differences arising from the hexagonal crystal symmetry of hBN and allow for a more accurate comparison. For all devices, $L=1 \mu \mathrm{m}$ and the contact length $L_{\mathrm{c}}=0.5 \mu \mathrm{m}$. Scale bars in $\mathbf{c}, \mathbf{h}$ : $10 \mu \mathrm{m}$.

Thus, our work introduces a universal approach for making efficient contacts to encapsulated $2 \mathrm{D}$ semiconductors, especially those sensitive to air, and marks an important step towards pristine devices with homogeneous electrical and optical characteristics on a macroscopic scale. We believe that with further improvement of the edge contact interface, by minimizing disorder and passivating in-gap edge states, as discussed later, even smaller $R_{\mathrm{C}}$ is achievable.

\section{Edge contacts fabrication}

We will now discuss the fabrication strategy that we developed. Detailed process parameters can be found in Supporting Section $\mathrm{S} 1$. Bottom hBN flakes were exfoliated directly on $p^{+} \mathrm{Si} / \mathrm{SiO}_{2}$ $(100 \mathrm{~nm})$ substrates. $1 \mathrm{~L}-\mathrm{MoS}_{2}$ and top $\mathrm{hBN}$ flakes were separately exfoliated on GelPak ${ }^{\circledR}$ PDMS (poly-dimethylsiloxane) stamps and transferred sequentially onto a suitable bottom hBN flake, as illustrated schematically in Figs. 11a, b. We found that PDMS can leave substantial residues behind after transfer which we minimized by pre-cleaning the PDMS surface in ultraviolet-ozone $\left(\mathrm{UV}-\mathrm{O}_{3}\right)$ prior to exfoliation (see Ref. 51 for details). After each transfer, the resulting stack was annealed at $200{ }^{\circ} \mathrm{C}$ in high-vacuum for $3 \mathrm{~h}$ to release trapped bubbles, wrinkles and strain (if any) induced by PDMS during transfer ${ }^{51}$. Figure $11 \mathrm{c}$ shows the optical image of a $1 \mathrm{~L}-\mathrm{MoS}_{2}$ flake transferred onto hBN from PDMS. To fully encapsulate the $\mathrm{MoS}_{2}$, another hBN flake was subsequently transferred on top.

For device fabrication, bubble-free areas were chosen and patterned into rectangular sections by e-beam lithography (EBL) with PMMA (poly-methylmethacrylate) and reactive ion etching (RIE). Contact trenches were defined in a second EBL step and the exposed $\mathrm{hBN}-\mathrm{MoS}_{2}-\mathrm{hBN}$ was etched away by RIE to create $\mathrm{MoS}_{2}$ edges for making contacts, as depicted in Fig. 11d (also see Supporting Fig. S1). The samples were then loaded into an e-beam evaporator for metal deposition, which we found to be the most critical part of the whole fabrication process. An etched $\mathrm{MoS}_{2}$ edge consists of dangling bonds as well as defects like Moand $\mathrm{S}$-vacancies that are much more reactive than the basal plane of $\mathrm{MoS}_{2}{ }^{52}$. During the time elapsed between etching and metal deposition, $\mathrm{O}_{2}$ and $\mathrm{H}_{2} \mathrm{O}$ molecules can not only bind to such edge sites but also potentially convert unpassivated $\mathrm{Mo}$ into $\mathrm{MoO}_{\mathrm{x}}{ }^{52}$. However, $\mathrm{MoO}_{\mathrm{x}}$, which is often used as a hole transport layer in solar cells, hinders electron injection into $\mathrm{MoS}_{2}$ due to its high work-function ${ }^{53}$. This scenario is in strong contrast to top contacts where $\mathrm{MoO}_{\mathrm{x}}$ formation is unlikely.

Hence, immediately before metal deposition, $\mathrm{MoO}_{\mathrm{x}}$ and any adsorbed $\mathrm{O}_{2}$ or $\mathrm{H}_{2} \mathrm{O}$ were removed by in-situ $\mathrm{Ar}^{+}$sputtering at $\pm 15^{\circ}$ tilt angle to expose a fresh $\mathrm{MoS}_{2}$ edge (Fig. 11e). Tilting the sample is necessary to access the etched $\mathrm{hBN}-\mathrm{MoS}_{2}-\mathrm{hBN}$ sidewalls shadowed by an overhanging PMMA bilayer with an inward slope and avoid re-deposition of sputtered PMMA over the $\mathrm{MoS}_{2}$ edges. Ti-Au $(5-40 \mathrm{~nm})$ was then deposited at $\pm 15^{\circ}$ tilt under a base pressure of $1 \times 10^{-7} \mathrm{mbar}$ (Fig. 11f-g). After lift-off, the devices were annealed in $\mathrm{Ar}+\mathrm{H}_{2}$ at $300{ }^{\circ} \mathrm{C}$ for $3 \mathrm{hrs}$ to improve the $\mathrm{Ti}-\mathrm{MoS}_{2}$ edge interface and reduce contact resistance (Supporting Section S2). Note that the use of Ti is essential for providing good adhesion to hBN sidewalls. Without Ti, pure Au tends to reflow and lose contact during annealing at $300{ }^{\circ} \mathrm{C}$ (Supporting Section $\mathrm{S} 4$ ). The final set of devices with edge contacts are shown in Fig. 11h.

\section{Electrical characterization}

Figure 22a shows the $I_{\mathrm{D}}-V_{\mathrm{DS}}$ output characteristics of an edge contacted $1 \mathrm{~L}-\mathrm{MoS}_{2}$ transistor exhibiting n-type behavior. A slight non-linearity at low $V_{\mathrm{DS}}$ indicates the presence of a small barrier 

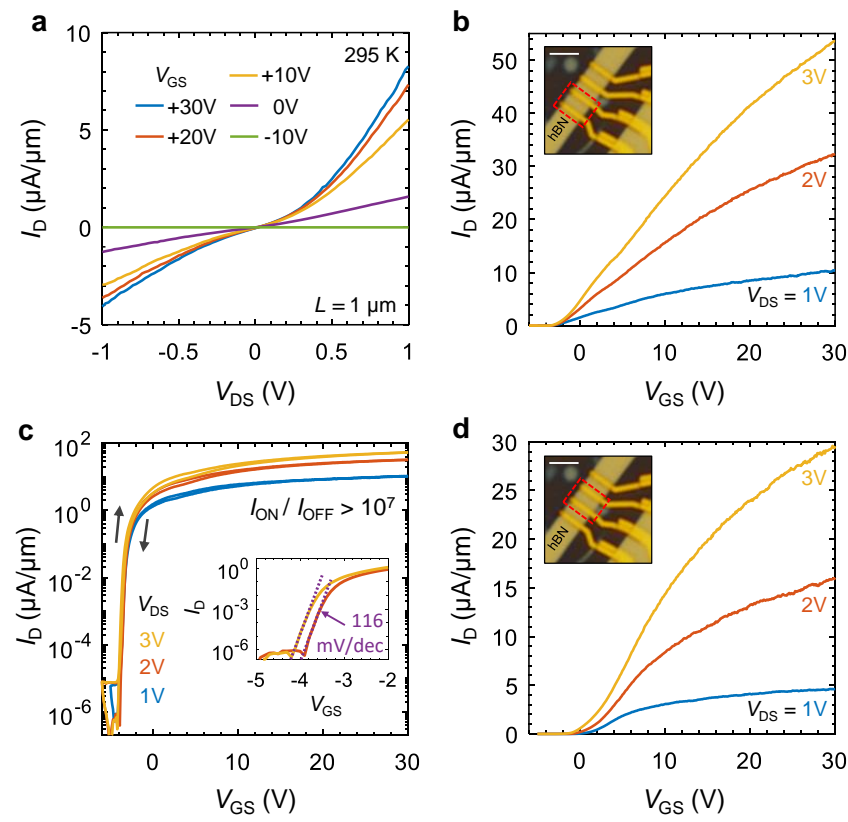

Figure 2. I-V measurements of monolayer $\mathrm{MoS}_{2}$ with edge contacts (a) $V_{\mathrm{GS}}$ dependent two-probe $I_{\mathrm{D}}-V_{\mathrm{DS}}$ characteristics of a $1 \mathrm{~L}-\mathrm{MoS}_{2}$ transistor measured under ambient conditions. (b) $I_{\mathrm{D}}-V_{\mathrm{GS}}$ characteristics of the same device demonstrating that edge contacts can support a high current density comparable to top contact devices with similar channel lengths but large metal-MoS 2 overlap areas ${ }^{21,54}$. Inset: Optical image of the measured device (outlined). (c) The data from $\mathbf{b}$ with $I_{\mathrm{D}}$ plotted on a log-scale showing both forward and backward sweeps to highlight the low hysteresis. Inset: Magnified plot of the subthreshold characteristics at $V_{\mathrm{DS}}=2 \mathrm{~V}, 3 \mathrm{~V}$ (only forward sweeps) exhibiting a steep slope and $<1 \mathrm{pA} / \mu \mathrm{m}$ off current. (d) $I_{\mathrm{D}}$ $V_{\mathrm{GS}}$ characteristics of the next device (inset) showing current magnitudes similar to the first device in $\mathbf{b}$. The devices presented in these figures were etched with $\mathrm{CHF}_{3}+\mathrm{O}_{2}$ and sputtered with $\mathrm{Ar}^{+}$before metal deposition to form edge contacts. Scale bars in $\mathbf{b}, \mathbf{d}: 3 \mu \mathrm{m}$.

at the contacts, as predicted by our quantum transport simulations (Supporting Section S6) and other computational studies ${ }^{55,56}$. The $I_{\mathrm{D}}-V_{\mathrm{GS}}$ transfer characteristics of the same device are plotted in Figs. 22b-c on linear and log scales, respectively. A high current density reaching $53.5 \mu \mathrm{A} / \mu \mathrm{m}$ at $V_{\mathrm{DS}}=3 \mathrm{~V}$ with an on-off ratio $>10^{7}$ can be observed. This clearly demonstrates that an efficient carrier injection is achievable via edge contacts, despite the lack of a $2 \mathrm{D}$ overlap between $\mathrm{MoS}_{2}$ and Ti. In Fig. 22c, each curve is comprised of both forward and backward sweeps which display a very small hysteresis. A magnified plot of the subthreshold characteristics is shown in the inset of Fig. 22c and reveals a low subthreshold swing (SS) of $116 \mathrm{mV} / \mathrm{dec}$ maintained up to nearly 4 orders of magnitude. Realization of such a steep slope and low hysteresis was made possible here by encapsulation in $\mathrm{hBN}$ which not only protects the $\mathrm{MoS}_{2}$ channel from processing residues, but also provides an atomically smooth dielectric interface free of dangling bonds and defects. This significantly decreases the interface trap density in comparison with an exposed $\mathrm{MoS}_{2}$ layer on a $\mathrm{SiO}_{2}$ substrate. Moreover, the absence of thermally populated surface optical phonons in hBN at room temperature leads to a reduced scattering rate and enhanced carrier mobilities in $\mathrm{MoS}_{2}{ }^{57}$. Using the relation ${ }^{58}$,

$$
\mathrm{SS}=(\ln 10) \frac{k_{\mathrm{B}} T}{q}\left(1+\frac{C_{\mathrm{it}}}{C_{\mathrm{G}}}\right)
$$

where $C_{\mathrm{G}}=27.8 \mathrm{nF} \mathrm{cm}^{-2}$ is the gate capacitance per unit area and $C_{\mathrm{it}}=q^{2} D_{\mathrm{it}}$ is the interface capacitance per unit area, we estimated the density of interface trap states $D_{\mathrm{it}}=1.7 \times 10^{11} \mathrm{eV}^{-1} \mathrm{~cm}^{-2}$. This value is at least an order of magnitude lower than for unencapsulated, lithographically exposed $\mathrm{MoS}_{2}$ on $\mathrm{SiO}_{2} 59,60$ and $\mathrm{ZrO}_{2}{ }^{1}$. Note that in this sample, the SS is primarily limited by the back-gate dielectric thickness $(100 \mathrm{~nm} \mathrm{SiO} 2+24 \mathrm{~nm} \mathrm{hBN})$. From Eq. (1), it is evident that for a larger gate capacitance (thinner dielectric), a smaller SS approaching the room temperature thermionic limit of $\sim 60 \mathrm{mV} / \mathrm{dec}$ is anticipated. The $I_{\mathrm{D}}-V_{\mathrm{GS}}$ characteristics of a second device on the same sample are plotted in Fig. 22d, displaying a current density comparable to Fig. 22b.

To investigate the influence of the metal- $\mathrm{MoS}_{2}$ contact edge cleanliness on carrier injection, a set of three control devices that were not $\mathrm{Ar}^{+}$sputtered, were also fabricated on the same $\mathrm{hBN}-\mathrm{MoS}_{2}-\mathrm{hBN}$ stack shown in Fig. 11h (outlined in red). All control devices conduct a significantly lower $I_{\mathrm{D}}$ than in Figs. 22b-d, indicating that carrier injection can be hindered if the $\mathrm{MoS}_{2}$ edge is not freshly cleaned immediately before metal deposition (Supporting Section S5). Additionally, it has been reported that Ti can partially oxidize during evaporation, depending on the vacuum level inside the deposition chamber, and thereby result in $\mathrm{TiO}_{\mathrm{x}}$ formation at the contact interface ${ }^{61}$. To inhibit the oxidation of $\mathrm{Ti}$, we deposited $\mathrm{Ti}$-Au on another set of devices (again without $\mathrm{Ar}^{+}$sputtering) at a $10 \mathrm{x}$ lower base pressure of $\sim 1 \times 10^{-8} \mathrm{mbar}$, with negligible residual $\mathrm{O}_{2}$ (Supporting Section S5). However, a low $I_{\mathrm{D}}$ is also observed in this case, revealing the existence of an $R_{\mathrm{C}}$ dominated transport. This implies that a better vacuum does not lead to any appreciable change in the contact properties if $\mathrm{Ar}^{+}$ sputtering is not done. It must be emphasized that an optimum post-deposition annealing temperature is also crucial for improving the contact interface (Supporting Section S2). Hence, our key finding here is that a clean $\mathrm{MoS}_{2}$ edge (before metallization) and annealing (after metallization) are both essential for forming good edge contacts, like those demonstrated in Figs. 22b-d. This likely explains why such a high current density had not been observed previously 48,49 .

Next, we want to characterize the intrinsic carrier mobility $\left(\mu_{0}\right)$ and contact resistance $\left(R_{\mathrm{C}}\right)$ of our devices. For an ideal long-channel nMOSFET operating in the strong inversion regime, a linear dependence of $I_{\mathrm{D}}$ on $V_{\mathrm{GS}}$ is expected, given by

$$
I_{\mathrm{D}}=\mu_{0} C_{\mathrm{G}} \frac{W}{L}\left(V_{\mathrm{GS}, \text { int }}-V_{\mathrm{T}}-V_{\mathrm{DS}, \text { int }} / 2\right) V_{\mathrm{DS}, \text { int }}
$$

where $V_{\mathrm{T}}$ is the threshold voltage and the internal drain $\left(V_{\mathrm{DS}, \text { int }}\right)$ and gate $\left(V_{\mathrm{GS}, \text { int }}\right)$ voltages are equal to the externally applied bias. Typically, $\mu_{0}$ is extracted from the slope of linear $I_{\mathrm{D}}-V_{\mathrm{GS}}$ characteristics with the help of Eq. (2). However, Fig. 22b shows that $I_{\mathrm{D}}$ grows sub-linearly with $V_{\mathrm{GS}}$ for all $V_{\mathrm{DS}}$, which causes the mobility extracted in this manner to be underestimated. For a more accurate description of such $I_{\mathrm{D}}-V_{\mathrm{GS}}$ behavior, the presence of finite contact resistances $R_{\mathrm{C}}$ in series with the $\mathrm{MoS}_{2}$ channel must be considered. In this scenario, the internal voltages seen by the channel get reduced to $V_{\mathrm{DS} \text {,int }}=V_{\mathrm{DS}}-2 I_{\mathrm{D}} R_{\mathrm{C}}$ and $V_{\mathrm{GS} \text {,int }}=V_{\mathrm{GS}}-I_{\mathrm{D}} R_{\mathrm{C}}$. Note that $V_{\mathrm{T}}$ also gets modified to $V_{\mathrm{T}}+\delta R_{\mathrm{C}} V_{\mathrm{GS}}$ but the drain-induced barrier lowering (DIBL) factor $\delta$ is small enough to be neglected in our long-channel devices. Equation (2) can then be re-written as

$$
I_{\mathrm{D}}=\mu_{0} C_{\mathrm{G}} \frac{W}{L}\left[\left(V_{\mathrm{GS}}-I_{\mathrm{D}} R_{\mathrm{C}}\right)-V_{\mathrm{T}}-\left(V_{\mathrm{DS}}-2 I_{\mathrm{D}} R_{\mathrm{C}}\right) / 2\right]\left(V_{\mathrm{DS}}-2 I_{\mathrm{D}} R_{\mathrm{C}}\right)
$$

Rearranging Eq. (3) to solve for $I_{\mathrm{D}}$, we obtain

$$
\begin{gathered}
\Longrightarrow I_{\mathrm{D}}=\frac{\mu_{0} C_{\mathrm{G}}}{1+\theta\left(V_{\mathrm{GS}}-V_{\mathrm{T}}-V_{\mathrm{DS}} / 2\right)} \frac{W}{L}\left(V_{\mathrm{GS}}-V_{\mathrm{T}}-V_{\mathrm{DS}} / 2\right) V_{\mathrm{DS}} \\
\text { where } \theta=2 R_{\mathrm{C}} \mu_{0} C_{\mathrm{G}} \frac{W}{L}
\end{gathered}
$$

From Eq. (4), we can infer that when $R_{\mathrm{C}} \neq 0, I_{\mathrm{D}}$ increases sub-linearly with $V_{\mathrm{GS}}$. To exclude the effect of $R_{\mathrm{C}}$, one can first calculate $1 / \sqrt{g_{\mathrm{m}}}$, as shown by Ghibaudo ${ }^{62}$ and $\mathrm{Jain}^{63}$, where $g_{\mathrm{m}} \equiv \partial I_{\mathrm{D}} / \partial V_{\mathrm{GS}}$ is the transconductance of the device.

$$
\frac{1}{\sqrt{g_{\mathrm{m}}}}=\left(\frac{L}{\mu_{0} C_{\mathrm{G}} V_{\mathrm{DS}} W}\right)^{1 / 2}\left[1+\theta\left(V_{\mathrm{GS}}-V_{\mathrm{T}}-V_{\mathrm{DS}} / 2\right)\right]
$$


a

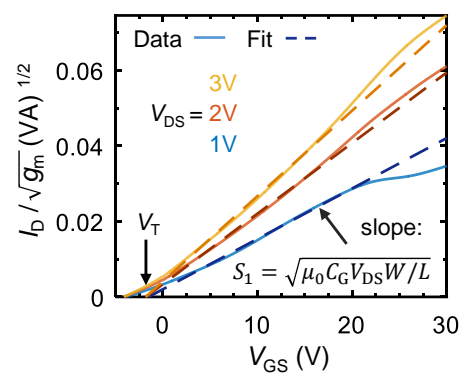

d

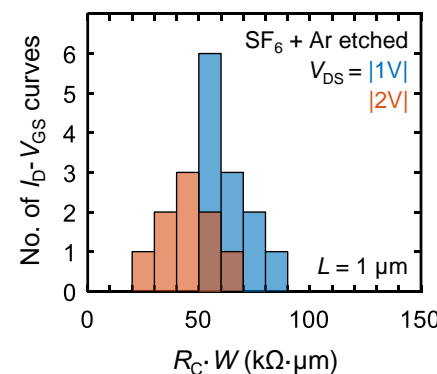

b

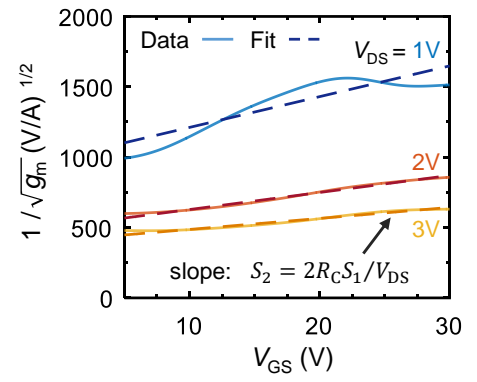

C

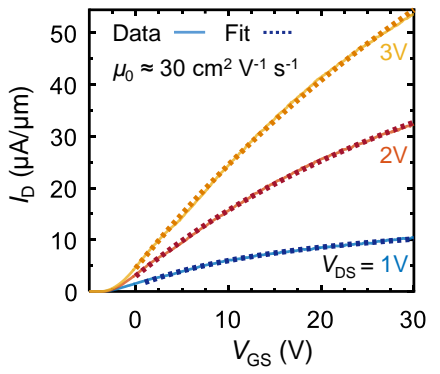

e

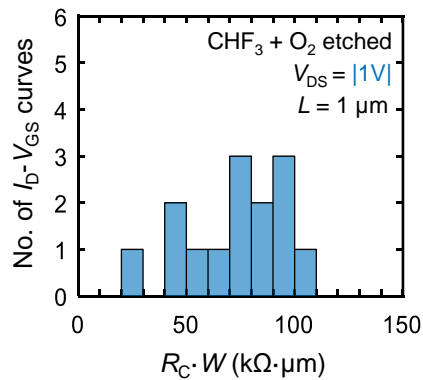

Figure 3. Mobility and contact resistance estimation. (a) $Y$-function $v s$. $V_{\mathrm{GS}}$ plot of the data shown in Fig. 22b. The dashed straight lines are fits to the linear region from which the mobility $\left(\mu_{0}\right)$ and threshold voltage $\left(V_{\mathrm{T}}\right)$ can be extracted using the slope $\left(S_{1}\right)$ and x-intercept, respectively. (b) $1 / \sqrt{g_{\mathrm{m}}} v s$. $V_{\mathrm{GS}}$ plot of the same dataset as a. The edge contact resistance can be estimated from the slopes $\left(S_{2}\right)$ of the linear fits using the given expression. (c) The $I_{\mathrm{D}}-V_{\mathrm{GS}}$ data from Fig. 22b fitted with the model in Eq. (4) choosing $\mu_{0}, \theta$ and $V_{\mathrm{T}}$ as fit parameters. The goodness of the fit confirms that Eq. (4) can accurately model our $I_{\mathrm{D}}-V_{\mathrm{GS}}$ characteristics. $\mu_{0} \approx 30 \mathrm{~cm}^{2} \mathrm{~V}^{-1} \mathrm{~s}^{-1}, R_{\mathrm{C}} \cdot W=27.8,11.7$ and $8.3 \mathrm{k} \Omega \cdot \mu \mathrm{m}$ at $V_{\mathrm{DS}}=1,2$ and $3 \mathrm{~V}$ were estimated from the fits for this device. (d) Histogram of $R_{\mathrm{C}} \cdot \mathrm{W}$ values for six devices etched with $\mathrm{SF}_{6}+$ Ar. For each device, the $R_{\mathrm{C}}$ was extracted by fitting the respective $I_{\mathrm{D}}-V_{\mathrm{GS}}$ curves with Eq. (4). The histogram includes $R_{\mathrm{C}}$ for both bias polarities $\left(V_{\mathrm{DS}}=+1 \mathrm{~V},-1 \mathrm{~V}\right.$ ) owing to slightly asymmetric $I_{\mathrm{D}}-V_{\mathrm{DS}}$ curves (see Fig. $22 \mathrm{a}$ ). In some cases, $R_{\mathrm{C}}$ was also extracted from $I_{\mathrm{D}}-V_{\mathrm{GS}}$ curves recorded at $V_{\mathrm{DS}}=|2 \mathrm{~V}|$ (orange bars). (e) Histogram of $R_{\mathrm{C}} \cdot \mathrm{W}$ values at $V_{\mathrm{DS}}=|1 \mathrm{~V}|$ for seven devices etched with $\mathrm{CHF}_{3}+\mathrm{O}_{2}$. An increased variability in $R_{\mathrm{C}}$ can be seen with $\mathrm{CHF}_{3}+\mathrm{O}_{2}$ compared to $\mathrm{SF}_{6}+\mathrm{Ar}$.

Upon multiplying Eqs. (4) and (5), $\theta$ can be eliminated and an expression commonly known as the $Y$-function is obtained, which depends linearly on $V_{\mathrm{GS}}$.

$$
Y \equiv \frac{I_{\mathrm{D}}}{\sqrt{g_{\mathrm{m}}}}=\left(\mu_{0} C_{\mathrm{G}} V_{\mathrm{DS}} \frac{W}{L}\right)^{1 / 2}\left(V_{\mathrm{GS}}-V_{\mathrm{T}}-V_{\mathrm{DS}} / 2\right)
$$

By plotting $Y$ vs. $V_{\mathrm{GS}}$ and using Eq. (6), the mobility $\left(\mu_{0}\right)$ and threshold voltage $\left(V_{\mathrm{T}}\right)$ can be extracted from the slope $\left(S_{1}\right)$ and $\mathrm{x}$-intercept, respectively ${ }^{13,64}$. Figure $33 \mathrm{a}$ is a plot of the $Y$-function for the data in Fig. 22b. It shows an approximately linear behaviour in the strong inversion regime from which a value of $\mu_{0}=29.2 \mathrm{~cm}^{2} \mathrm{~V}^{-1} \mathrm{~s}^{-1}$ can be derived. Lastly, we plot $1 / \sqrt{g_{\mathrm{m}}}$ vs. $V_{\mathrm{GS}}$ (Fig. 33b) and extract the slope $\left(S_{2}\right)$ of the linear region. $R_{\mathrm{C}}$ can then be determined from the relation $R_{\mathrm{C}}=S_{2} V_{\mathrm{DS}} / 2 S_{1}$, derived using Eqs. (4)-(6) ${ }^{65}$. However, we found that due to random undulations of the derivative $\partial I_{\mathrm{D}} / \partial V_{\mathrm{GS}}$, the plots in Fig. 33b do not always remain linear in the entire $V_{\mathrm{GS}}-V_{\mathrm{T}}>V_{\mathrm{DS}} / 2$ range for every device. As a consequence, the extracted slope can vary depending on the range chosen for the 1D polynomial fit. Hence, for a more reliable estimation of device parameters, we followed a slightly different approach and directly fitted our $I_{\mathrm{D}}-V_{\mathrm{GS}}$ curves with Eq. (4), choosing all three unknowns $\left(V_{\mathrm{T}}, \theta\right.$ and $\left.\mu_{0}\right)$ as fitting parameters. We found this procedure to be more straightforward than the commonly used $Y$-function method.

Figure $33 \mathrm{c}$ is a reproduction of the plots in Fig. 22b, fitted with Eq. (4) in the inversion regime for each $V_{\mathrm{DS}}$. The excellent quality of the fits indicates that the model in Eq. (4) describes our $I_{\mathrm{D}}-V_{\mathrm{GS}}$ characteristics very well. The estimated mobility $\mu_{0}=$ $29.8 \mathrm{~cm}^{2} \mathrm{~V}^{-1} \mathrm{~s}^{-1}$ is also in good agreement with the value obtained from the $Y$-function plots in Fig. 33a. Knowing $\mu_{0}$ and $\theta$ from the fits, the contact resistance could then be deduced using Eq. (4) to be $R_{\mathrm{C}} \cdot W=27.8,11.7$ and $8.3 \mathrm{k} \Omega \cdot \mu \mathrm{m}$ at $V_{\mathrm{DS}}=1,2$ and $3 \mathrm{~V}$, respectively. It was found to decrease with increasing $V_{\mathrm{DS}}$ owing to enhanced Schottky barrier tunneling at higher bias voltages, as indicated by the non-linear $I_{\mathrm{D}}-V_{\mathrm{DS}}$ characteristics in Fig. 22a.
Interestingly, these numbers are very similar to room temperature $R_{\mathrm{C}}$ values reported for graphene top contacts on hBN encapsulated $1 \mathrm{~L}-\mathrm{MoS}_{2}$ devices $(20 \mathrm{k} \Omega \cdot \mu \mathrm{m})^{28}$ as well as CVD grown lateral graphene $-\mathrm{MoS}_{2}$ contacts $(10-50 \mathrm{k} \Omega \cdot \mu \mathrm{m})^{37,38}$. But at the same time, compared to the latter case, we observe a higher mobility owing to $\mathrm{hBN}$ encapsulation. These results unambiguously demonstrate that edge contacts can replace graphene contacts in encapsulated devices and achieve better performance with a less restrictive and scalable fabrication methodology. Moreover, use of graphene with $\mathrm{MoS}_{2}$ is currently limited to electron injection only, whereas with a proper choice of edge contact material, hole injection can also be feasible ${ }^{55}$.

For completeness, it should be clarified that in our analysis $R_{\mathrm{C}}$ is assumed to be independent of $V_{\mathrm{GS}}$ whereas in conventional contacts, it decreases asymptotically with increasing carrier density for low $V_{\mathrm{GS}}$ near the onset of inversion, and slowly saturates at high $V_{\mathrm{GS}}$. Such a behavior arises from the fact that in Ti-MoS 2 top contacts with an interfacial oxide (often unintentional), increasing $V_{\mathrm{GS}}$ reduces the sheet resistivity of $\mathrm{MoS}_{2}$ below the contact region and also lowers the potential barrier for electron injection into $\mathrm{MoS}_{2}{ }^{66}$, which increases the effective current transfer length $L_{\mathrm{T}}{ }^{67}$. Moreover, at the same time the applied $V_{\mathrm{GS}}$ also pushes the conduction band (CB) minimum closer to the metal Fermi level, thereby bending the $\mathrm{CB}$ more steeply near the contact edge, which narrows the effective Schottky barrier width ${ }^{67}$. This two-fold mechanism leads to a strong reduction of $R_{\mathrm{C}}$ in top contacts as $V_{\mathrm{GS}}$ increases ${ }^{68}$. However, in edge contacts where a $2 \mathrm{D}$ metal- $\mathrm{MoS}_{2}$ overlap region is absent $\left(L_{\mathrm{T}} \approx 0\right)$, the primary mechanism behind $R_{\mathrm{C}}$ reduction with increasing $V_{\mathrm{GS}}$ is Schottky barrier narrowing, being more pronounced near the subthreshold region and saturating soon after. This causes $R_{\mathrm{C}}$ to show a gate dependence that is weak enough to be neglected for $V_{\mathrm{GS}}-V_{\mathrm{T}} \gg V_{\mathrm{DS}} / 2$, as substantiated by the constant slopes $S_{1}$ and $S_{2}$ in Figs. 33a-b for $V_{\mathrm{GS}}>5 \mathrm{~V}$, which justifies our initial assumption. The model in Eq. (4) also fits well only in this regime. Hence, the $R_{\mathrm{C}}$ we estimated is the $V_{\mathrm{GS}}$ independent value at large carrier densities, similar to Ref. 64 . The most accurate way of extracting $R_{\mathrm{C}}$ is the transfer length method (TLM). However, 


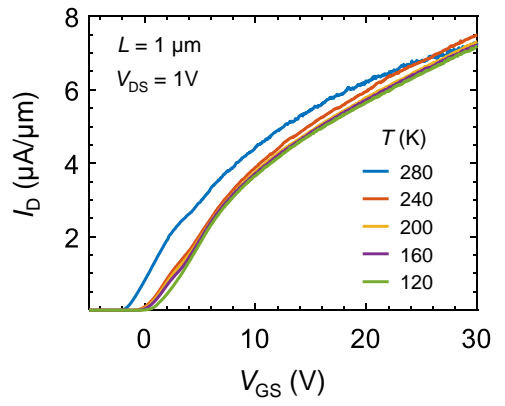

b

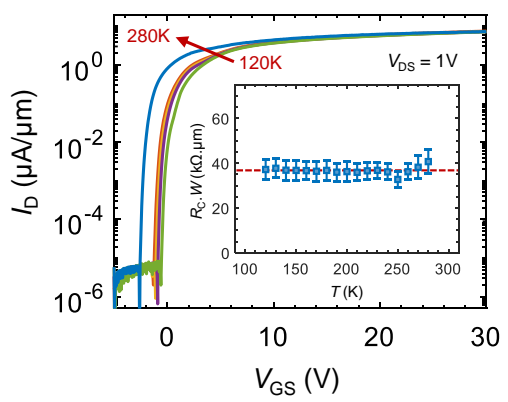

C

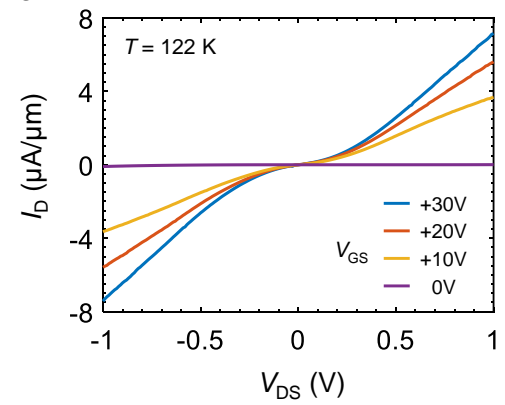

Figure 4. Low temperature measurements. $I_{\mathrm{D}}-V_{\mathrm{GS}}$ characteristics of an edge contacted $1 \mathrm{~L}-\mathrm{MoS}$ transistor at various temperatures plotted on (a) linear and (b) $\log$ scales. Inset: Extracted $R_{\mathrm{C}}$ values revealing a temperature independent behavior, which indicates that edge contacts can perform well even at low temperatures. The error bars denote $99 \%$ confidence intervals of the fitted values. The horizontal red dashed line is a guide to the eye. (c) $I_{\mathrm{D}}-V_{\mathrm{DS}}$ curves at $122 \mathrm{~K}$ displaying similar characteristics as at room temperature (Fig. 22a).

it requires fabrication of several devices with decreasing channel lengths and only works well when all devices have a very similar $R_{\mathrm{C}}$ and $\mu_{0}$, such that a plot of total resistance $v s$. channel length follows a straight line. This has turned out to be challenging at present for our devices. Hence, we employed a simpler method, which gives a reasonable estimate of $R_{\mathrm{C}}$ and $\mu_{0}$ for every single device and also helps in quantifying the device-to-device variability, unlike TLM.

Besides the device in Fig. 33c, we obtained similarly good fits for $I_{\mathrm{D}}-V_{\mathrm{GS}}$ curves measured from additional devices, which further corroborates the model we used (see Supporting Section $\mathrm{S} 3$ for more $I-V$ datasets). From these fits, an average $\mu_{0}=$ $(20.5 \pm 5.5) \mathrm{cm}^{2} \mathrm{~V}^{-1} \mathrm{~s}^{-1}$ was found, where the error margin represents one standard deviation. To study the influence of etched $\mathrm{hBN}$ sidewall profiles on edge contacts, we tested two different $\mathrm{hBN}$ etch recipes ${ }^{46,69}$. Figures 33d-e are histograms of $R_{\mathrm{C}}$ extracted from devices etched using the two recipes. For $\mathrm{SF}_{6}+\mathrm{Ar}$ etched devices, we estimate an average $R_{\mathrm{C}} \cdot W=(64.2 \pm 9.6) \mathrm{k} \Omega \cdot \mu \mathrm{m}$ at $V_{\mathrm{DS}}=|1 \mathrm{~V}|$ (blue bars). Since our $I_{\mathrm{D}}-V_{\mathrm{DS}}$ curves are slightly asymmetric in general (Fig. 22a), we extracted $R_{\mathrm{C}}$ values from $I_{\mathrm{D}}-V_{\mathrm{GS}}$ fits for both positive and negative $V_{\mathrm{DS}}$. Some devices were also measured at $V_{\mathrm{DS}}$ $=|2 \mathrm{~V}|$ (orange bars) with an average $R_{\mathrm{C}} \cdot W=(46 \pm 10) \mathrm{k} \Omega \cdot \mu \mathrm{m}$. In contrast to $\mathrm{SF}_{6}+\mathrm{Ar}$ etched devices, those etched with $\mathrm{CHF}_{3}+\mathrm{O}_{2}$ show a wider distribution (Fig. 33e) and a higher mean value of $R_{\mathrm{C}} \cdot W=(73.5 \pm 23.4) \mathrm{k} \Omega \cdot \mu \mathrm{m}$. We attribute this increased variability to greater etching inhomogeneity resulting from $\mathrm{CHF}_{3}+\mathrm{O}_{2}$ in comparison with $\mathrm{SF}_{6}+\mathrm{Ar}$, which we discovered upon scanning electron microscopy of bare hBN sidewalls (Supporting Fig. S1).

We further characterized another edge contacted $1 \mathrm{~L}-\mathrm{MoS}_{2}$ device at low temperatures inside a liquid nitrogen filled cryostat and is presented in Fig. 44. Apart from the expected shift in threshold voltage $V_{\mathrm{T}}$ to higher values ${ }^{58}$, we find that the $I_{\mathrm{D}}-V_{\mathrm{GS}}$ characteristics as well as the edge contact resistance in Figs. 44a-b remain essentially unchanged up to $120 \mathrm{~K}$. This observed temperature insensitivity of $R_{\mathrm{C}}$ agrees very well with previous findings on $1 \mathrm{D}$ edge contacts to graphene ${ }^{46}$ as well as CVD grown graphene edge contacts to $1 \mathrm{~L}-\mathrm{MoS}_{2}{ }^{37}$, and demonstrates that carrier injection into $\mathrm{MoS}_{2}$ via edge contacts occurs efficiently even under cryogenic conditions. Moreover, the $I_{\mathrm{D}}-V_{\mathrm{DS}}$ characteristics at $122 \mathrm{~K}$ plotted in Fig. $44 \mathrm{c}$, behave similar to those at room temperature seen earlier for the device in Fig. 22a. To shed some light on this behavior, we performed $a b$ initio quantum transport simulations following the procedure described in our earlier publication ${ }^{66}$ and are discussed in Supporting Section S6. In brief, the majority of the current injected via Ti edge contacts into $\mathrm{MoS}_{2}$ does not come from thermionic emission over the contact Schottky barrier, but rather tunneling across the barrier. Since the electron transmission probability near the Fermi level remains relatively constant as a function of energy (Supporting Fig. S10), the tunneling current varies only weakly with temperature. We found that this tendency persists for a range of Schottky barrier heights that were evaluated.

\section{Discussion and conclusions}

Strictly speaking, the true bandstructure of a semiconductor is defined for a lattice with an infinitely repeating unit cell. At $\mathrm{MoS}_{2}$ edges and grain boundaries, dangling bonds and Mo-, S-vacancies perturb the $\mathrm{MoS}_{2}$ bandstructure and give birth to additional localized 'edge states' within the bandgap, as measured experimentally ${ }^{11,70}$. Such states were also observed in air-exposed $\mathrm{MoS}_{2}{ }^{11}$ and $\mathrm{WSe}_{2}{ }^{71}$ devices, implying that adsorbed $\mathrm{O}_{2}$ and $\mathrm{H}_{2} \mathrm{O}$ do not fully passivate them. Passivation of dangling bonds and edge states is essential for good edge contacts ${ }^{72}$, which may be achieved by Ti-MoS 2 bonding. However, if a van der Waals gap or trapped air molecules are present between the $\mathrm{MoS}_{2}$ edge and Ti, edge state passivation could be hindered, resulting in a high density of in-gap states at each electron injection site. By trapping incoming electrons, these states can cause a space charge region to build up which would repel further injected electrons. In this regard, in-situ $\mathrm{Ar}^{+}$sputtering plays a key role in producing a clean $\mathrm{MoS}_{2}$ edge immediately before Ti deposition. Subsequent annealing at $300^{\circ} \mathrm{C}$ promotes atomic rearrangement and $\mathrm{Ti}-\mathrm{MoS}_{2}$ bonding. The need for such extra measures does not arise in the case of edge contacts to graphene, where edge states (if any) are unable to trap carriers because of the absence of a bandgap. Even $\mathrm{O}_{2}$ incorporation at the graphene edge was shown to have a negligible effect ${ }^{46}$, thus greatly simplifying fabrication of edge contacts to graphene. This scenario is fundamentally different from top contacts where the injected electrons do not encounter any edge states since the translational symmetry of the underlying $\mathrm{MoS}_{2}$ lattice is not broken (in the absence of interfacial reactions and defects) and on the contrary, a vdW gap is beneficial for avoiding Fermi level pinning ${ }^{34}$.

Interactions between the contact metal and $\mathrm{MoS}_{2}$ at the atomic scale and structural characteristics of the contact interface play a significant role in governing the performance of any contact. For edge contacts in particular, where carrier transfer is restricted to a single atomic edge, an optimum metal- $\mathrm{MoS}_{2}$ interface is crucial. This makes them more challenging to fabricate compared to top contacts which impose fewer constraints and can tolerate local non-idealities to a greater extent due to the availability of a finite area. Our main achievement here lies in the development of an optimized process for realizing low resistance edge contacts with a high density of current injection per atomic site. Further studies are needed nevertheless to unravel the rich physics and chemistry occurring at the contact interface. Atomically resolved cross-sectional transmission electron microscope (TEM) imaging can be performed to gain better insights into the contact morphology, interface quality and atomic configuration of edge contacts. This would lead to a deeper understanding of the transport behavior and provide valuable guidelines for further improvement of the contact performance. It is possible that unpassivated edge states at interface voids still undermine the performance of our devices $^{72}$ and also cause undesired Fermi level pinning ${ }^{73}$. Suitable chemical termination of dangling bonds could be a promising 
strategy to passivate edge states, de-pin the metal Fermi level and reduce $R_{\mathrm{C}}$ even further. Apart from $\mathrm{MoS}_{2}$, air sensitive TMDCs like $\mathrm{HfS}_{2}, \mathrm{ZrS}_{2}$, etc. where edge states are expected to lie at shallow levels close to the band extrema, which makes them more immune to defects, appear as attractive materials for edge contacts ${ }^{74}$.

It should be emphasized that even though $\sim \mu \mathrm{m}$ long $\mathrm{Ti}-\mathrm{Au}^{54}$, $\mathrm{Ag}-\mathrm{Au}^{13}$ and $\mathrm{In}-\mathrm{Au}^{75}$ top contacts on $1 \mathrm{~L}-\mathrm{MoS}_{2}$ have resulted in a lower $R_{\mathrm{C}}$ than that obtained in this work, in order to be fair, a comparison should be made with top contacts scaled down to sub-nm overlap lengths. However, it has been shown that the $R_{\mathrm{C}}$ begins to increase considerably for contact lengths smaller than the current transfer length in both mono- ${ }^{67}$ and multi-layer $\mathrm{MoS}_{2}{ }^{76}$. This implies that conventional contacts cannot be scaled down beyond a certain limit, thereby restricting the minimum achievable device footprint (gate length $+2 \mathrm{x}$ contact length). To ensure scaling of TMDC based devices, scalable contact geometries that work efficiently irrespective of dimensions are necessary. This bottleneck could be overcome by means of edge contacts, which do not require a 2D overlap with TMDCs and thus, in principle, can be made as narrow as possible. Another domain where edge contacts can outperform top contacts is multilayer TMDCs, in which carrier injection only via the topmost layer suffers from added interlayer hopping resistances that limit the current transport to top few layers ${ }^{77}$, whereas with edge contacts, each layer can be individually contacted for achieving higher current densities ${ }^{78}$. In this regard, 1T-phase edge contacts to few layer $\mathrm{MoS}_{2}{ }^{79}$ and $\mathrm{MoTe}_{2}{ }^{80}$ also seem to be an attractive choice, although inducing a $2 \mathrm{H} \rightarrow 1 \mathrm{~T}$ phase transition under the contact regions after encapsulation can be problematic.

Lastly, the possibility to encapsulate $2 \mathrm{D}$ materials before processing with chemicals remains the biggest advantage of edge contacts for building clean devices. Fundamental studies rely on high interface quality and macroscopic homogeneity for uncovering new physical phenomena, which can benefit from edge contacts fabricated after encapsulation. Edge contacts are especially promising for 2D materials unstable in air for which fabrication of top contacts is challenging due to restrictions imposed by encapsulation inside an inert atmosphere before being exposed to air. Often such heterostructures are built in a top-down manner and the need to make contacts to buried layers demands pick-up of additional graphene sheets. In such scenarios, edge contacts provide a much higher flexibility in heterostructure assembly and can be scaled-up to integrated circuits employing multiple metal layers separated by insulating dielectric layers. Thus, we envision that edge contacts will bring devices based on 2D materials one step closer to practical implementation and open up new pathways in 2D materials research.

\section{Associated Content}

Supporting Information. Detailed description of the fabrication procedure, plots showing the influence of annealing, data from additional devices, pure Au edge contacts without $\mathrm{Ti}$, edge contacts without $\mathrm{Ar}^{+}$sputtering, quantum transport simulations.

\section{Methods}

Edge contact fabrication. See Supporting Section S1 for a step-by-step process flow.

Electrical characterization. I-V measurements were carried out using a Keithley 2602B source meter in two-probe configuration. All devices were measured in air at room temperature (except those shown in Fig. 44). For calculating $g_{m}$, the $I_{\mathrm{D}}-V_{\mathrm{GS}}$ curves were smoothened by cubic spline interpolation in MATLAB to reduce the noise before differentiation.

\section{Acknowledgments}

This research was supported by the Swiss National Science Foundation (grant no. 200021_165841), ETH Zürich (ETH-32 15-1) and CSCS (Project s876). Use of the cleanroom facilities at the FIRST Center for Micro and Nanoscience, ETH Zürich is gratefully acknowledged. TT and KW acknowledge support from the Elemental Strategy Initiative conducted by the MEXT, Japan and JSPS KAKENHI (grant no. JP15K21722). AJ would like to thank Aroosa Ijaz for invaluable help during sample fabrication.

\section{Author Contributions}

$\mathrm{ML}, \mathrm{AJ}$ and $\mathrm{LN}$ conceived the project. AJ developed the fabrication procedure, carried out the measurements and analyzed the experimental data ÁS and ML performed the quantum transport simulations. MP built the electrical characterization setup, wrote the LabVIEW scripts for recording $\mathrm{I}-\mathrm{V}$ data and provided experimental support at various stages. Low temperature transport measurements were performed together with EB. TT and KW synthesized the hBN crystals used in this study. LN, ML and PB supervised the project. AJ wrote the manuscript with inputs from MP, ML and LN. acjain@ethz.ch

lnovotny@ethz.ch

1 Desai, S. B. et al. $\mathrm{MoS}_{2}$ transistors with 1-nanometer gate lengths. Science 354, 99-103 (2016)

2 Iannaccone, G., Bonaccorso, F., Colombo, L. \& Fiori, G. Quantum engineering of transistors based on 2D materials heterostructures. Nature Nanotech. 13, 183-191 (2018).

3 Frisenda, R., Molina-Mendoza, A. J., Mueller, T., Castellanos-Gomez, A. \& van der Zant, H. S. J. Atomically thin p-n junctions based on two-dimensional materials. Chem. Soc. Rev. 47, 3339-3358 (2018).

4 Wang, M. et al. Robust memristors based on layered two-dimensional materials. Nature Electron. 1, 130-136 (2018).

5 Bharadwaj, P. \& Novotny, L. Optoelectronics in flatland. Optics and Photonics News 26, 24-31 (2015).

6 Wang, J., Verzhbitskiy, I. \& Eda, G. Electroluminescent devices based on 2D semiconducting transition metal dichalcogenides. Adv. Mater. 30, 1802687 (2018).

7 Schaibley, J. R. et al. Valleytronics in 2D materials. Nat. Rev. Mater. 1, 16055 (2016).

8 Qiu, H. et al. Electrical characterization of back-gated bi-layer $\mathrm{MoS}_{2}$ field-effect transistors and the effect of ambient on their performances. Appl. Phys. Lett. 100, 98-101 (2012).

9 Jariwala, D. et al. Band-like transport in high mobility unencapsulated single-layer $\mathrm{MoS}_{2}$ transistors. Appl. Phys. Lett. 102, 4-8 (2013).

10 Park, W. et al. Oxygen environmental and passivation effects on molybdenum disulfide field effect transistors. Nanotechnology 24, 095202
(2013).

$11 \mathrm{Wu}, \mathrm{D}$. et al. Uncovering edge states and electrical inhomogeneity in $\mathrm{MoS}_{2}$ field-effect transistors. Proc. Natl. Acad. Sci. USA 113, 8583-8588 (2016).

12 Rahimi, S. et al. The positive effects of hydrophobic fluoropolymers on the electrical properties of $\mathrm{MoS}_{2}$ transistors. Appl. Sci. 6, 236 (2016).

13 Smithe, K. K. H., Suryavanshi, S. V., Muñoz Rojo, M., Tedjarati, A. D. \& Pop, E. Low variability in synthetic monolayer $\mathrm{MoS}_{2}$ devices. ACS Nano 11, 8456-8463 (2017).

14 Giannazzo, F., Fisichella, G., Piazza, A., Agnello, S. \& Roccaforte, F. Nanoscale inhomogeneity of the Schottky barrier and resistivity in $\mathrm{MoS}_{2}$ multilayers. Phys. Rev. B 92, 081307 (2015).

$15 \mathrm{Ji}$, H. et al. Gas adsorbates are coulomb scatterers, rather than neutral ones, in a monolayer $\mathrm{MoS}_{2}$ field effect transistor. Nanoscale 10, 10856-10862 (2018).

16 Sangwan, V. K. et al. Low-frequency electronic noise in single-layer $\mathrm{MoS}_{2}$ transistors. Nano Letters 13, 4351-4355 (2013).

17 Xie, X. et al. Low-frequency noise in bilayer $\mathrm{MoS}_{2}$ transistor. ACS Nano 8, 5633-5640 (2014).

18 Shimazu, Y., Tashiro, M., Sonobe, S. \& Takahashi, M. Environmental effects on hysteresis of transfer characteristics in molybdenum disulfide field-effect transistors. Sci. Rep. 6, 6-11 (2016).

19 Cho, K. et al. Gate-bias stress-dependent photoconductive characteristics of multi-layer $\mathrm{MoS}_{2}$ field-effect transistors. Nanotechnology 25, 155201 (2014).

20 Baugher, B., Churchill, H. O. H., Yang, Y. \& Jarillo-Herrero, P. Intrinsic 
electronic transport properties of high quality monolayer and bilayer $\mathrm{MoS}_{2}$. Nano Lett. 13, 4212-4216 (2013).

21 Smithe, K. K., English, C. D., Suryavanshi, S. V. \& Pop, E. Intrinsic electrical transport and performance projections of synthetic monolayer $\mathrm{MoS}_{2}$ devices. 2D Materials 4, 1-8 (2017).

22 Peto, J. et al. Spontaneous doping of the basal plane of $\mathrm{MoS}_{2}$ single layers through oxygen substitution under ambient conditions. Nature Chem. 10, 1246-1251 (2018).

${ }^{23}$ Lee, G.-H. et al. Highly stable, dual-gated $\mathrm{MoS}_{2}$ transistors encapsulated by hexagonal boron nitride with gate-controllable contact resistance and threshold voltage. ACS Nano 9, 7019-7026 (2015).

24 Park, J. H. et al. Scanning tunneling microscopy and spectroscopy of air exposure effects on molecular beam epitaxy grown $\mathrm{WSe}_{2}$ monolayers and bilayers. ACS Nano 10, 4258-4267 (2016).

25 Mirabelli, G. et al. Air sensitivity of $\mathrm{MoS}_{2}, \mathrm{MoSe}_{2}, \mathrm{MoTe}_{2}, \mathrm{HfS}_{2}$, and $\mathrm{HfSe}_{2}$. J. Appl. Phys. 120, 125102 (2016).

26 Gao, J. et al. Aging of transition metal dichalcogenide monolayers. ACS Nano 10, 2628-2635 (2016).

27 Cao, Y. et al. Quality heterostructures from two-dimensional crystals unstable in air by their assembly in inert atmosphere. Nano Lett. 15 , 4914-4921 (2015).

28 Cui, X. et al. Multi-terminal transport measurements of $\mathrm{MoS}_{2}$ using a van der Waals heterostructure device platform. Nature Nanotech. 10, 534-540 (2015).

29 Liu, Y. et al. Toward barrier free contact to molybdenum disulfide using graphene electrodes. Nano Lett. 15, 3030-3034 (2015).

30 Guan, J., Chuang, H. J., Zhou, Z. \& Tománek, D. Optimizing charge injection across transition metal dichalcogenide heterojunctions: Theory and experiment. ACS Nano 11, 3904-3910 (2017).

31 Sata, Y. et al. N- and p-type carrier injections into $\mathrm{WSe}_{2}$ with van der Waals contacts of two-dimensional materials. Jpn. J. Appl. Phys. 56, 04CK09 (2017).

32 Wang, J. I.-J. et al. Electronic transport of encapsulated graphene and $\mathrm{WSe}_{2}$ devices fabricated by pick-up of prepatterned hBN. Nano Lett. 15, 1898-1903 (2015).

33 Telford, E. J. et al. Via method for lithography free contact and preservation of 2D materials. Nano Lett. 18, 1416-1420 (2018).

34 Liu, Y. et al. Approaching the Schottky-Mott limit in van der Waals metal-semiconductor junctions. Nature 557, 696-700 (2018).

35 Liao, M. et al. Twist angle-dependent conductivities across $\mathrm{MoS}_{2}$ /graphene heterojunctions. Nature Coтmun. 9, 4068 (2018).

36 Ling, X. et al. Parallel stitching of 2D materials. Adv. Mater. 28, 2322 2329 (2016).

37 Guimarães, M. H. et al. Atomically thin Ohmic edge contacts between two-dimensional materials. ACS Nano 10, 6392-6399 (2016).

38 Zhao, M. et al. Large-scale chemical assembly of atomically thin transistors and circuits. Nature Nanotech. 11, 954-959 (2016).

39 Suenaga, K. et al. Surface-mediated aligned growth of monolayer $\mathrm{MoS}_{2}$ and in-plane heterostructures with graphene on sapphire. ACS Nano 12, 10032-10044 (2018).

${ }^{40}$ Han, Y. et al. Strain mapping of two-dimensional heterostructures with subpicometer precision. Nano Lett. 18, 3746-3751 (2018).

41 Wang, J. et al. High mobility $\mathrm{MoS}_{2}$ transistor with low Schottky barrier contact by using atomic thick h-BN as a tunneling layer. Adv. Mater. $\mathbf{2 8}$, 8302-8308 (2016).

42 Cui, X. et al. Low temperature Ohmic contact to monolayer $\mathrm{MoS}_{2}$ by van der Waals bonded Co/h-BN electrodes. Nano Lett. 17, 4781-4786 (2017).

$43 \mathrm{Li}, \mathrm{X}$. X. et al. Gate-controlled reversible rectifying behaviour in tunnel contacted atomically-thin $\mathrm{MoS}_{2}$ transistor. Nature Commun. 8 (2017).

44 Ghiasi, T. S., Quereda, J. \& van Wees, B. J. Bilayer h-BN barriers for tunneling contacts in fully-encapsulated monolayer $\mathrm{MoSe}_{2}$ field-effect transistors. 2D Materials 6, 015002 (2018).

45 Lee, S., Tang, A., Aloni, S. \& Philip Wong, H.-S. Statistical study on the Schottky barrier reduction of tunneling contacts to CVD synthesized $\mathrm{MoS}_{2}$. Nano Lett. 16, 276-281 (2016).

46 Wang, L. et al. One-dimensional electrical contact to a two-dimensional material. Science 342, 614-617 (2013).

47 Karpiak, B. et al. 1D ferromagnetic edge contacts to 2D graphene/h-BN heterostructures. 2D Materials 5, 014001 (2017).

48 Chai, Y. et al. Making one-dimensional electrical contacts to molybdenum disulfide-based heterostructures through plasma etching. Phys. Stat. Sol. A 213, 1358-1364 (2016).

49 Moon, B. H. et al. Junction-structure-dependent Schottky barrier inhomogeneity and device ideality of monolayer $\mathrm{MoS}_{2}$ field-effect transistors. ACS Appl. Mater. Interfaces 9, 11240-11246 (2017).

$50 \mathrm{Xu}$, S. et al. Universal low-temperature Ohmic contacts for quantum transport in transition metal dichalcogenides. 2D Materials 3, 021007 (2016).

51 Jain, A. et al. Minimizing residues and strain in 2D materials transferred from PDMS. Nanotechnology 29, 265203 (2018).

52 Martincová, J., Otyepka, M. \& Lazar, P. Is single layer $\mathrm{MoS}_{2}$ stable in the air? Chem. Eur. J 23, 13233-13239 (2017).

53 K. C., S., Longo, R. C., Addou, R., Wallace, R. M. \& Cho, K. Electronic properties of $\mathrm{MoS}_{2} / \mathrm{MoO}_{\mathrm{x}}$ interfaces: Implications in tunnel field effect transistors and hole contacts. Sci. Rep. 6, 33562 (2016).

${ }^{54}$ Liu, W., Sarkar, D., Kang, J., Cao, W. \& Banerjee, K. Impact of contact on the operation and performance of back-gated monolayer $\mathrm{MoS}_{2}$ fieldeffect-transistors. ACS Nano 9, 7904-7912 (2015).

55 Guo, Y., Liu, D. \& Robertson, J. 3D behavior of Schottky barriers of 2D transition-metal dichalcogenides. ACS Appl. Mater. Interfaces 7, 25709-25715 (2015).

56 Dong, W. \& Littlewood, P. B. Quantum electron transport in Ohmic edge contacts between two-dimensional materials. ACS Appl. Electron. Mater. 1, 799-803 (2019)

57 Dean, C. R. et al. Boron nitride substrates for high-quality graphene electronics. Nature Nanotech. 5, 722-726 (2010).

58 Sze, S. M. \& Ng, K. K. Physics of Semiconductor Devices, 3rd Edition (John Wiley \& Sons, 2006).

59 Zou, X. et al. Interface engineering for high-performance top-gated $\mathrm{MoS}_{2}$ field-effect transistors. Adv. Mater. 26, 6255-6261 (2014).

${ }^{60}$ Choi, K. et al. Trap density probing on top-gate $\mathrm{MoS}_{2}$ nanosheet field-effect transistors by photo-excited charge collection spectroscopy. Nanoscale 7, 5617-5623 (2015).

${ }^{61}$ McDonnell, S., Smyth, C., Hinkle, C. L. \& Wallace, R. M. MoS 2 -titanium contact interface reactions. ACS Appl. Mater. Interfaces 8, 8289-8294 (2016).

62 Ghibaudo, G. New method for the extraction of MOSFET parameters. Electron. Lett. 24, 543-545 (1988).

63 Jain, S. Measurement of threshold voltage and channel length of submicron MOSFETs. IEE Proc. I - Solid-State Electron Devices 135, 162-164 (1988).

64 Chang, H. Y., Zhu, W. \& Akinwande, D. On the mobility and contact resistance evaluation for transistors based on $\mathrm{MoS}_{2}$ or two-dimensional semiconducting atomic crystals. Appl. Phys. Lett. 104 (2014).

65 Cho, K. et al. Contact-engineered electrical properties of $\mathrm{MoS}_{2}$ fieldeffect transistors via selectively deposited thiol-molecules. Adv. Mater. 30, 1705540 (2018).

66 Szabó, A., Jain, A., Parzefall, M., Novotny, L. \& Luisier, M. Electron

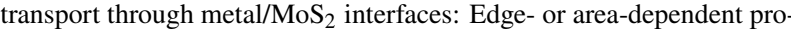
cess? Nano Lett. 19, 3641-3647 (2019).

${ }^{67} \mathrm{Liu}$, H. et al. Switching mechanism in single-layer molybdenum disulfide transistors: An insight into current flow across Schottky barriers. ACS Nano 8, 1031-1038 (2014).

68 Allain, A., Kang, J., Banerjee, K. \& Kis, A. Electrical contacts to twodimensional semiconductors. Nature Mat. 14, 1195-1205 (2015).

69 Autore, M. et al. Boron nitride nanoresonators for phonon-enhanced molecular vibrational spectroscopy at the strong coupling limit. Light Sci. Appl. 7, 17172 (2018).

${ }^{70}$ Bollinger, M. V. et al. One-dimensional metallic edge states in $\mathrm{MoS}_{2}$ Phys. Rev. Lett. 87, 196803 (2001).

71 Addou, R. et al. One dimensional metallic edges in atomically thin $\mathrm{WSe}_{2}$ induced by air exposure. 2D Materials 5, 025017 (2018).

72 Houssa, M. et al. Contact resistance at graphene/MoS 2 lateral heterostructures. Appl. Phys. Lett. 114, 163101 (2019).

73 Chen, W., Yang, Y., Zhang, Z. \& Kaxiras, E. Properties of in-plane graphene/MoS 2 heterojunctions. 2D Materials 4, 045001 (2017).

74 Pandey, M. et al. Defect-tolerant monolayer transition metal dichalcogenides. Nano Lett. 16, 2234-2239 (2016).

75 Wang, Y. et al. Van der Waals contacts between three-dimensional metals and two-dimensional semiconductors. Nature 568, 70-74 (2019).

76 English, C. D., Shine, G., Dorgan, V. E., Saraswat, K. C. \& Pop, E. Improved Contacts to $\mathrm{MoS}_{2}$ Transistors by Ultra-High Vacuum Metal Deposition. Nano Lett. 16, 3824-3830 (2016).

77 Das, S. \& Appenzeller, J. Where does the current flow in two-dimensional layered systems? Nano Lett. 13, 3396-3402 (2013).

78 Schulman, D. S., Arnold, A. J. \& Das, S. Contact engineering for 2D materials and devices. Chem. Soc. Rev. 47, 3037-3058 (2018).

79 Kappera, R. et al. Phase-engineered low-resistance contacts for ultrathin $\mathrm{MoS}_{2}$ transistors. Nature Mat. 13, 1128-1134 (2014).

${ }^{80}$ Sung, J. H. et al. Coplanar semiconductor-metal circuitry defined on few-layer $\mathrm{MoTe}_{2}$ via polymorphic heteroepitaxy. Nature Nanotech. 12, 1064-1070 (2017). 


\title{
Supporting Information
}

\section{One-dimensional edge contacts to a monolayer semiconductor}

\author{
Achint Jain, ${ }^{1,}$ * Áron Szabó, ${ }^{2}$ Markus Parzefall, ${ }^{1}$ Eric Bonvin, ${ }^{1}$ Takashi Taniguchi, ${ }^{3}$ \\ Kenji Watanabe, ${ }^{3}$ Palash Bharadwaj, ${ }^{4}$ Mathieu Luisier, ${ }^{2}$ and Lukas Novotny ${ }^{1, \dagger}$ \\ ${ }^{1}$ Photonics Laboratory, ETH Zürich, 8093 Zürich, Switzerland \\ ${ }^{2}$ Integrated Systems Laboratory, ETH Zürich, 8092 Zürich, Switzerland \\ ${ }^{3}$ National Institute for Material Science, 1-1 Namiki, Tsukuba 305-0044, Japan \\ ${ }^{4}$ Department of Electrical and Computer Engineering, Rice University, Houston, TX 77005, USA
}

\section{List of Figures}

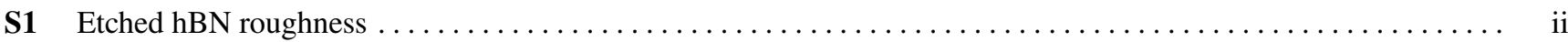

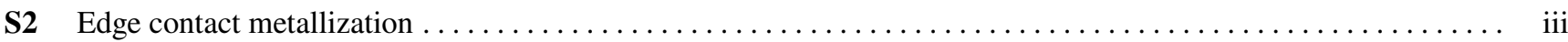

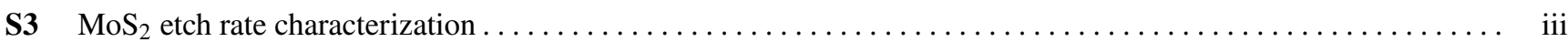

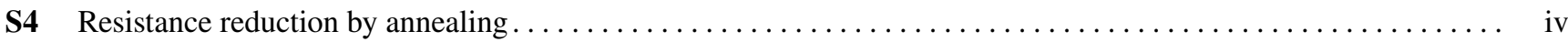

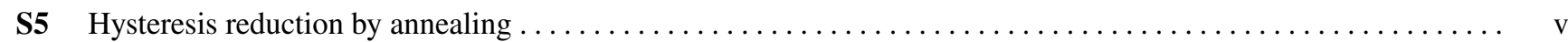

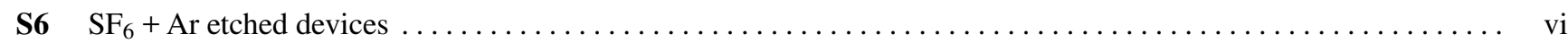

S7 Pure Au edge contacts $\ldots \ldots \ldots \ldots \ldots \ldots \ldots \ldots \ldots \ldots \ldots \ldots \ldots \ldots \ldots \ldots \ldots \ldots \ldots \ldots \ldots \ldots \ldots \ldots \ldots \ldots$

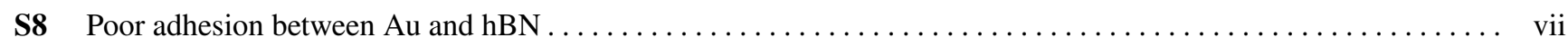

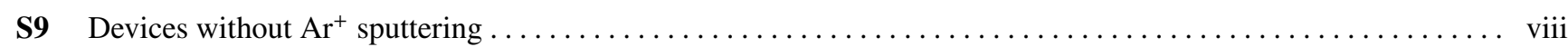

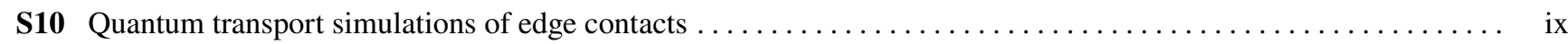




\section{S1. Fabrication details}

hBN-MoS - -hBN heterostructure assembly: Bottom hBN flakes were directly exfoliated on $\mathrm{O}_{2}$ plasma cleaned $p^{+} \mathrm{Si} / \mathrm{SiO}{ }_{2}$ $(100 \mathrm{~nm})$ substrates using a blue tape (Nitto). Naturally occurring $\mathrm{MoS}_{2}$ crystals (SPI Supplies) were exfoliated on viscoelastic PDMS stamps (Gel-Film ${ }^{\circledR}$ PF-40-X4 sold by Gel-Pak ${ }^{\circledR}$ ). We found that PDMS often has a significant amount of uncrosslinked (or 'loosely bound') dimethylsiloxane oligomers on its surface ${ }^{1}$ that can contaminate 2D materials exfoliated on PDMS ${ }^{2}$ In order to achieve high carrier mobilities and avoid unintentional doping, it is crucial to minimize PDMS residues and transfer $\mathrm{MoS}_{2}$ in a pristine manner. Therefore, prior to $\mathrm{MoS}_{2}$ exfoliation, all PDMS stamps were treated with UV-O 3 in a Bioforce Nanosciences UV-ozone ProCleaner for $30 \mathrm{~min}$ (manufacturer specified illumination intensity: $14.76 \mathrm{~mW} / \mathrm{cm}^{2}$ ), following the procedure outlined in ref. 2. $\mathrm{UV}-\mathrm{O}_{3}$ exposure breaks down unwanted surface oligomers and forms a residue free, few $\sim$ nm thin $\mathrm{SiO}_{\mathrm{x}}$ layer on the PDMS surface ${ }^{3}$. After $\mathrm{UV}_{-} \mathrm{O}_{3}$ treatment, PDMS stamps were left in ambient air for $2 \mathrm{~h}$ to deactivate the surface termination and cause a partial hydrophobic recovery of the PDMS surface. This wait interval prevents bonding between PDMS and the blue tape and helps to increase the yield during exfoliation.

Bulk $\mathrm{MoS}_{2}$ crystals were then exfoliated on the clean PDMS stamps after $2 \mathrm{~h}$ and monolayer flakes were identified in an optical microscope. For transfer, PDMS stamps with $\mathrm{MoS}_{2}$ were placed on a transparent quartz plate and aligned on top of suitable bottom hBN flakes on $\mathrm{SiO}_{2}$ using a SÜSS MicroTec MJB4 mask aligner. All transfers were carried out in air. Upon coming in contact, the $\mathrm{hBN}-\mathrm{MoS}_{2}$ stacks were heated to $\sim 65^{\circ} \mathrm{C}$ for 2 min with a Peltier module kept underneath the $\mathrm{Si} / \mathrm{SiO}{ }_{2}$ substrates. After allowing for a few minutes to cool down, the PDMS stamps were then slowly detached. The transferred MoS $\mathrm{M}_{2}$ lakes on hBN were annealed at $200^{\circ} \mathrm{C}$ for $3 \mathrm{~h}$ in high vacuum $\left(<1 \times 10^{-5} \mathrm{mbar}\right)$ to remove any remaining PDMS residues and release transfer induced compressive strain as well as accumulated bubbles/wrinkles in $\mathrm{MoS}_{2}{ }^{2}$. The same procedure was followed to transfer the top hBN flakes onto the $\mathrm{MoS}_{2}$ - (bottom) hBN stacks and the resulting heterostructures were again vacuum annealed at $200{ }^{\circ} \mathrm{C}$ for $3 \mathrm{~h}$ to reduce the density of bubbles.

Electron beam lithography (EBL) and etching: Clean, bubble-free areas in the hBN-MoS $-\mathrm{hBN}$ heterostructures were chosen and patterned into well-defined rectangular channels by EBL (RAITH150 Two) with a bilayer of PMMA 50k (4\% in chlorobenzene) and 950k (4.5\% in anisole). Each layer was spin-coated at $5000 \mathrm{rpm}$ for $45 \mathrm{~s}$ and baked at $180{ }^{\circ} \mathrm{C}$ for $4 \mathrm{~min}$. After EBL (electron dose: $400 \mu \mathrm{C} / \mathrm{cm}^{2}$ at $30 \mathrm{kV}$ ), PMMA was developed in MIBK:IPA (1:3) for $60 \mathrm{~s}$ and the exposed areas were etched away by reactive ion etching (RIE) in an Oxford Plasmalab 80 Plus system. For making edge contacts into the rectangular channels, EBL and RIE were repeated again. RIE was performed with either (a) $\mathrm{CHF}_{3}+\mathrm{O}_{2}$ plasma $(40+4 \mathrm{sccm}, 50 \mathrm{~W}$ power and $37.5 \mathrm{mTorr}$ pressure, etch rate: $\sim 36 \mathrm{~nm} / \mathrm{min})^{4}$ or $(\mathbf{b}) \mathrm{SF}_{6}+$ Ar plasma $(20+20 \mathrm{sccm}, 50 \mathrm{~W}$ power and $100 \mathrm{mTorr}$ pressure, etch rate: $>90 \mathrm{~nm} / \mathrm{min})^{5}$. Scanning electron microscope $(\mathrm{SEM})$ images of the hBN surfaces resulting from the two etching recipes are shown in Fig. $\mathrm{S} 1$ for comparison and reveal some striking differences. Reactive ion etching of hBN with $\mathrm{CHF}_{3}+\mathrm{O}_{2}$ proceeds primarily via chemical interactions that strongly favor etching along certain in-plane crystal directions. It can be noticed in Fig. S1a that this highly anisotropic etch rate leaves behind a high density of hBN pyramids in the etched regions and forms sidewalls with very narrow triangular crevices (red dotted lines). It is reasonable to assume that during metal deposition over such hBN sidewalls, Ti grains might not fill these fine crevices entirely, resulting in a loss of contact with the MoS $\mathrm{M}_{2}$ edge at certain spots.

a

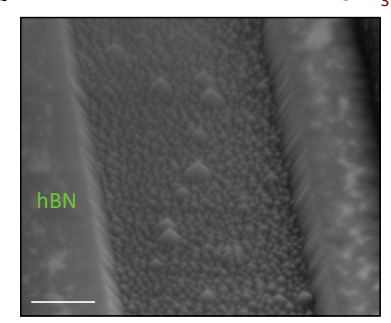

$\mathrm{CHF}_{3}+\mathrm{O}_{2}$

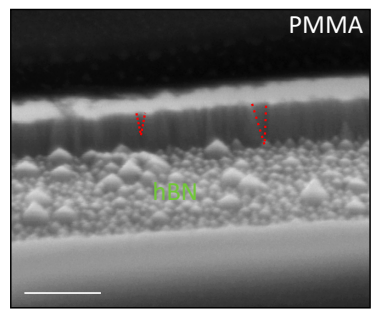

b

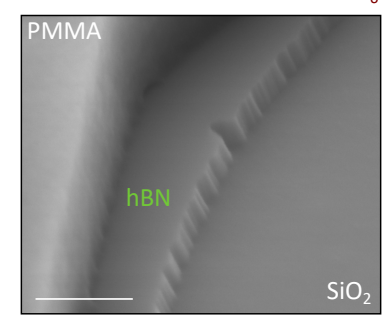

$\mathrm{SF}_{6}+\mathrm{Ar}$

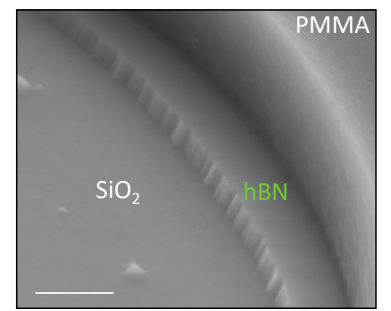

Figure S1. Etched hBN roughness. (a) Tilted view SEM images of contact trenches in a test hBN flake, etched half-way through with $\mathrm{CHF}_{3}+\mathrm{O}_{2}$ for $210 \mathrm{~s}$. The etched regions display a very rough bottom surface, with partially etched hBN pyramids, and sidewalls featuring narrow, vertical crevices with triangular facets, arising from the crystallographic planes of hBN. Two such crevices have been indicated by red dotted lines. (b) SEM images of a curved trench etched in another (thinner) hBN flake with $\mathrm{SF}_{6}+\mathrm{Ar}$ for $20 \mathrm{~s}$. It exhibits a smooth bottom $\mathrm{SiO}_{2}$ surface with a significantly reduced density of unetched hBN pyramids. No triangular crevices like those indicated in a could be resolved in this flake, although considerable sidewall roughness is still present. All images were taken immediately after etching, without removing PMMA, in order to avoid deposition of any organic residues that might smear out the sharp hBN features and reduce image contrast. Upon interaction with the electron beam $(5 \mathrm{keV})$, the PMMA layer retracted away from the sides, allowing $\mathrm{hBN}$ to be imaged. Scale bars: $200 \mathrm{~nm}$ in all images. 
Moreover, within a single device, source and drain contacts formed along parallel etched trenches would have different faceting due to the $60^{\circ}$ rotational symmetry of $\mathrm{hBN}$ (and not $90^{\circ}$ ), giving rise to asymmetric $I_{\mathrm{D}}-V_{\mathrm{DS}}$ characteristics. In case of $\mathrm{SF}_{6}+\mathrm{Ar}$ (1:1), the introduction of Ar adds a physical sputtering contribution to the etch process. This leads to more isotropic in-plane etching, resulting in sidewalls devoid of triangular facets, as shown in Fig. S1b and lower contact variability (see Fig. S6).

Metal deposition and annealing: This is the most critical part of our fabrication process. As discussed in the main text, etched $\mathrm{MoS}_{2}$ edges have a large number of dangling bonds that can host in-gap edge states ${ }^{6}$ and act as adsorption sites for air molecules $\left(\mathrm{O}_{2}, \mathrm{H}_{2} \mathrm{O}\right)$. These adsorbed species could likely hinder covalent bonding between the contact metal (Ti) and MoS 2 , leaving edge states unpassivated, which subsequently act as traps for injected carriers. Moreover, Mo atoms located at the edge can oxidize to form $\mathrm{MoO}_{\mathrm{x}}$ which, owing to its high work-function, would pose a further barrier for electron injection ${ }^{7}$. Therefore, in order to limit $\mathrm{MoS}_{2}$ oxidation after RIE, the samples were immediately loaded into an electron beam evaporator (Plassys MEB550S) for metal deposition, with only a few minutes of air exposure in between. While loading, the etched contact trenches were aligned parallel to the axis of the tilting motor and thereafter, the samples were not rotated in-plane at any point during sputtering and evaporation. To remove any $\mathrm{MoO}_{\mathrm{x}}$ and form a clean $\mathrm{MoS}_{2}$ edge just before metal deposition, the contact edges were sputtered in-situ with an Ar ${ }^{+}$ ion beam at $+15^{\circ}$ and $-15^{\circ}$ tilt (Fig. S2b) for $15 \mathrm{~s}$ each $(3.5 \mathrm{sccm}$ flow rate, $250 \mathrm{~V}$ beam voltage, $50 \mathrm{~V}$ acceleration voltage, $10 \mathrm{~mA}$ beam current). Since ion-gun parameters can vary from machine-to-machine, in order to make it easier for others to reproduce our recipe, we estimated the etch rate of bare $\mathrm{MoS}_{2}$ in our case by comparing the optical images in Fig. S3 before and after sputtering.

Ti $(5 \mathrm{~nm})$ was then deposited at $0.2 \mathrm{~nm} / \mathrm{s}$ rate under a base pressure of $1 \times 10^{-7}$ mbar, first at $+15^{\circ}$ tilt and then $5 \mathrm{~nm}$ again at $-15^{\circ}$ tilt, as depicted in Fig. S2c. Tilting was necessary to avoid shadowing of the $\mathrm{MoS}_{2}$ edge by the overhanging bilayer PMMA sidewalls. In this manner, a nearly conformal Ti layer could be deposited over the hBN sidewalls despite the rough topography, as visible in Fig. S2d. Au $(40+40 \mathrm{~nm})$ was then deposited at $0.2 \mathrm{~nm} / \mathrm{s}$ in the same way. Note that the choice of deposition angle was constrained by the PMMA undercut angle in our case. Deposition at $20^{\circ}$ caused the PMMA sidewalls to be also partially coated with $\mathrm{Ti}-\mathrm{Au}$, leaving behind vertical ears upon lift-off, while deposition at $30^{\circ}$ resulted in no lift-off. After lift-off in hot acetone, the samples were annealed at $300^{\circ} \mathrm{C}$ in $\mathrm{Ar}+\mathrm{H}_{2}(380+20 \mathrm{sccm})$ for $3 \mathrm{~h}$ inside a quartz tube furnace to improve the contacts. Finally, for electrical characterization, wire-bonds were made manually with a $25 \mu \mathrm{m}$ diameter tungsten wire and silver epoxy (CircuitWorks ${ }^{\circledR} \mathrm{CW} 2400$ ). The epoxy was cured at $80^{\circ} \mathrm{C}$ for 30 min in a vacuum oven. Unlike conventional ultrasonic wire-bonding, $\mathrm{Ag}$ epoxy helped to prevent shorting with the $\mathrm{Si}$ back-gate through the $100 \mathrm{~nm} \mathrm{SiO}_{2}$ layer.

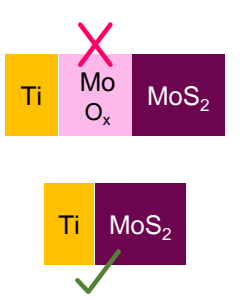

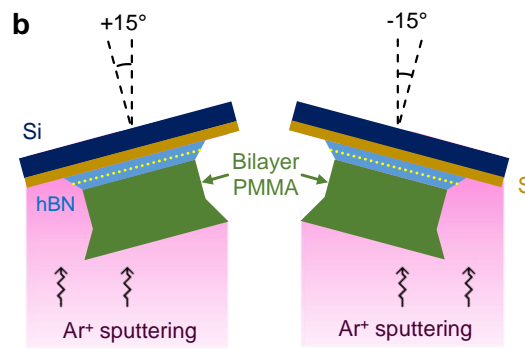

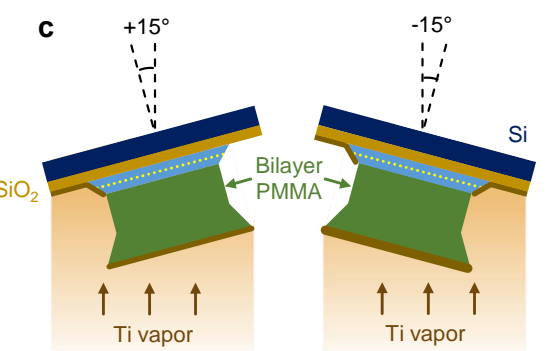

d

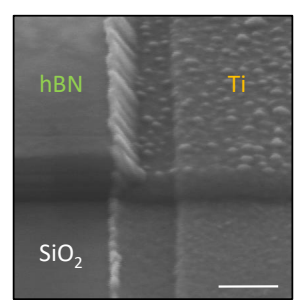

Figure S2. Edge contact metallization. (a) Sketch showing possible existence of $\mathrm{MoO}_{\mathrm{x}}$ at the Ti-MoS 2 interface (top) whereas a $\mathrm{MoO}$-free interface is desired (bottom). (b) Schematic illustration of an $\mathrm{hBN}-\mathrm{MoS}_{2}-\mathrm{hBN}$ heterostructure mounted upside-down inside an e-beam evaporator chamber, undergoing in-situ $\mathrm{Ar}^{+}$sputtering at $+15^{\circ}$ and $-15^{\circ}$ tilt to remove $\mathrm{MoO}_{\mathrm{x}}$ (if any) and absorbed $\mathrm{O}_{2}, \mathrm{H}_{2} \mathrm{O}$ molecules from both $\mathrm{MoS}_{2}$ contact edges before metal deposition. (c) Schematic illustration of Ti deposition at $+15^{\circ}$ and $-15^{\circ}$ tilt, immediately after $\mathrm{Ar}^{+}$sputtering. (d) SEM image of a test hBN flake featuring a conformal deposition of Ti over the $\mathrm{hBN}$ sidewall, despite its roughness. Scale bar: $100 \mathrm{~nm}$.

a

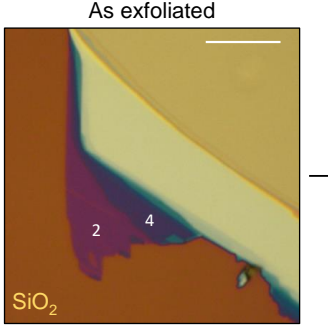

$\mathrm{Ar}^{+}$sputtered

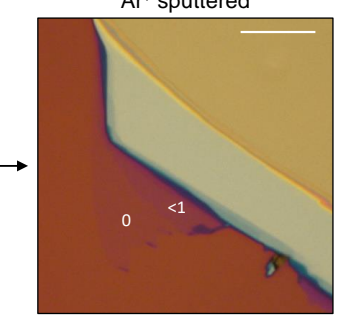

b

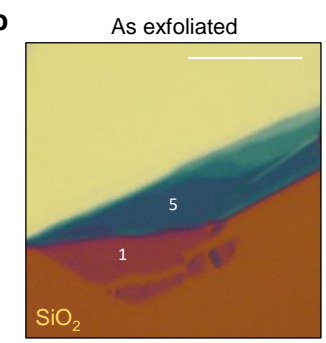

$\mathrm{Ar}^{+}$sputtered

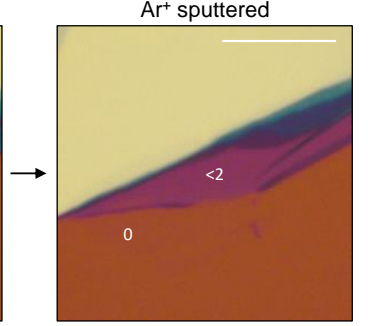

Figure S3. $\mathrm{MoS}_{2}$ etch rate characterization. Optical images of $\mathrm{MoS}_{2}$ flakes, as exfoliated on $\mathrm{Si}_{\mathrm{SiO}} \mathrm{S}_{2}(285 \mathrm{~nm})$ substrates, and after $\mathrm{Ar}{ }^{+}$ sputtering for $15 \mathrm{~s}$ (without tilting). The number of layers, before and after sputtering, have been indicated in all images from which an etch depth of $\sim 3.5$ layers in $15 \mathrm{~s}$ can be estimated. Scale bars: $10 \mu \mathrm{m}$ in all images. 


\section{S2. Significance of annealing}

Post metal-deposition annealing is commonly employed to lower the contact resistance $\left(R_{\mathrm{C}}\right)$ in $2 \mathrm{D}$ material devices. We tested several temperatures for this purpose and found that for edge contacts annealed in $\mathrm{Ar}+\mathrm{H}_{2}(380+20 \mathrm{sccm}), I_{\mathrm{D}} \mathrm{improved}$ remarkably with increasing temperatures up to $400{ }^{\circ} \mathrm{C}$, as shown in Fig. S4a. At the same time, the high interface quality of our devices remained preserved due to $\mathrm{hBN}$ encapsulation, as evidenced by the unchanged steep subthreshold slope and negligible hysteresis in all $I_{\mathrm{D}}-V_{\mathrm{GS}}$ plots in Fig. S4b. Besides this, we also varied the annealing time at a fixed temperature but did not notice a substantial change in $I_{\mathrm{D}}$ after a total duration $>1.5 \mathrm{~h}$ (Fig. S4c). We further extracted the $R_{\mathrm{C}}$ and mobility values from the curves in Fig. S4a and are plotted in Figs. S4d-e, respectively. A reduction in $R_{\mathrm{C}}$ and mobility enhancement at higher temperatures clearly highlight the importance of annealing. However, we also observed that too high temperatures can affect the long-term ambient stability of our devices to a certain extent. Considering these facts, a temperature of $300{ }^{\circ} \mathrm{C}$ and time $2-3 \mathrm{~h}$ were therefore chosen as optimum for our fabrication recipe. Lastly, un-annealed devices measured directly after metal deposition and lift-off, can sometimes exhibit a large hysteresis in the transfer characteristics, as seen in Fig. S5a. Annealing can help to get rid of such hysteresis (Fig. S5b).

Thus, we can conclude that annealing is indispensable for edge contacts. In addition to $\mathrm{Ar}+\mathrm{H}_{2}$, we also tested annealing of edge contacts in a high vacuum environment $\left(<1 \times 10^{-5} \mathrm{mbar}\right)$, but the former resulted in a slightly better performance. Several processes can occur simultaneously during annealing: metal-MoS $\mathrm{S}_{2}$ covalent bonding, reduction of interfacial oxides and sulfur atoms by $\mathrm{H}_{2}$ molecules, inter-diffusion of edge atoms (metal into $\mathrm{MoS}_{2}$ or vice-versa), defect migration and edge reconstruction. Presently, it is unclear what processes dominate in case of edge contacts and are responsible for the reduction of $R_{\mathrm{C}}$ upon annealing, although Fig. S4c points towards a weaker contribution of diffusion, which is a slow, time-dependent process. Understanding the various mechanisms at play and developing more effective annealing procedures is a promising subject for future studies.

a

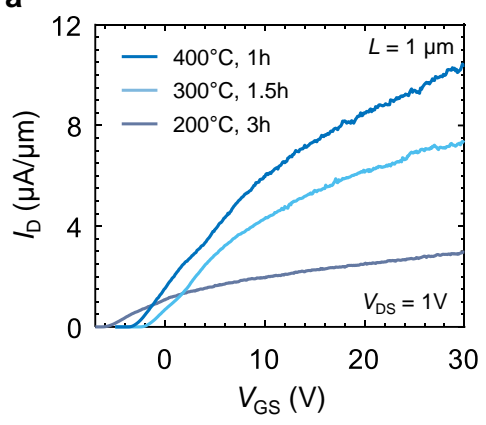

b

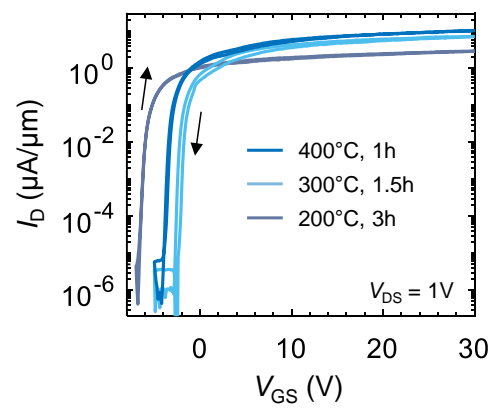

C

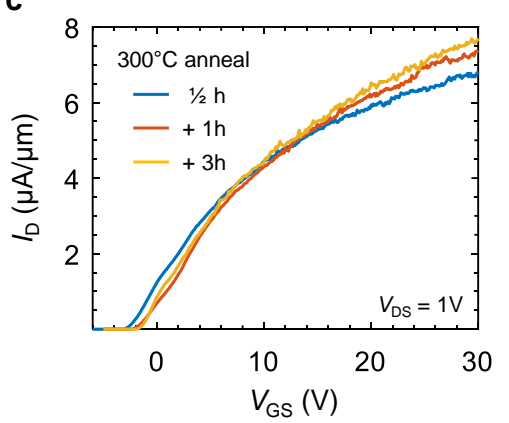

d

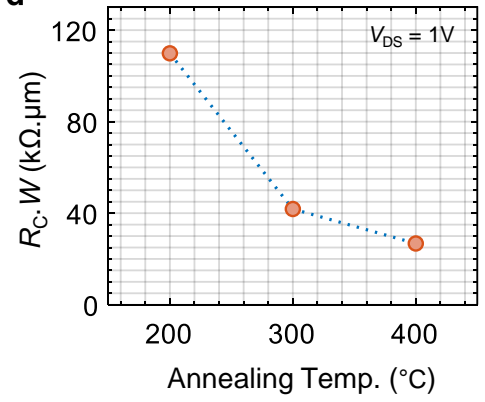

e

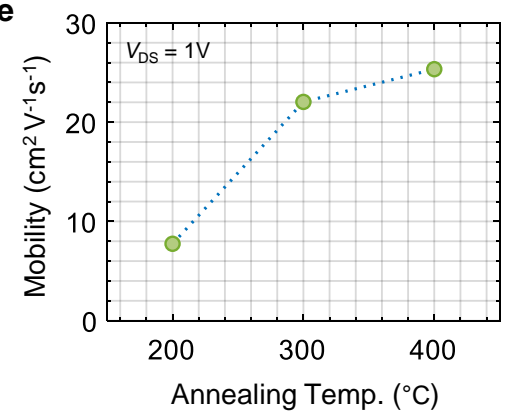

Figure S4. Contact resistance reduction by annealing. (a) $I_{\mathrm{D}}-V_{\mathrm{GS}}$ characteristics of a $1 \mathrm{~L}-\mathrm{MoS} 2$ device measured after annealing in Ar $+\mathrm{H}_{2}$ at three different temperatures. It can be seen that the current density increases monotonically with the annealing temperature, implying a reduction in $R_{\mathrm{C}}$ upon annealing. This enhancement can possibly be attributed to improvement of the edge contact interface, leading to reduced scattering/trapping of electrons and thus, more efficient current injection. (b) $I_{\mathrm{D}}-V_{\mathrm{GS}}$ data shown in a, plotted on a log-scale, exhibiting a consistently steep subthreshold slope after each annealing cycle. Moreover, all plots comprise of both forward and backward sweeps that display very small hysteresis (sweep directions marked by arrows). This indicates that in our devices, the $\mathrm{MoS}_{2}$ crystal quality and the low interface trap density $\left(\sim 10^{11} \mathrm{eV}^{-1} \mathrm{~cm}^{-2}\right)$, remain preserved by hBN encapsulation and do not deteriorate, at least up to $400^{\circ} \mathrm{C}$. (c) $I_{\mathrm{D}}-V_{\mathrm{GS}}$ characteristics of the same device after annealing at $300{ }^{\circ} \mathrm{C}$ for $30 \mathrm{~min}, 1 \mathrm{~h}$ and $3 \mathrm{~h}$, incrementally. These results reveal that prolonged annealing ( $>1.5 \mathrm{~h}$ ), at a fixed temperature, does not cause any significant change in the contact properties. (d-e) Contact resistance and mobility values extracted from the plots in a for each annealing temperature. Note: All data presented here was recorded from the same device shown in Figs. $2 \mathrm{a}-\mathrm{c}$ of the main text. After each annealing cycle, the sample was cooled down and measured at room temperature in air. 

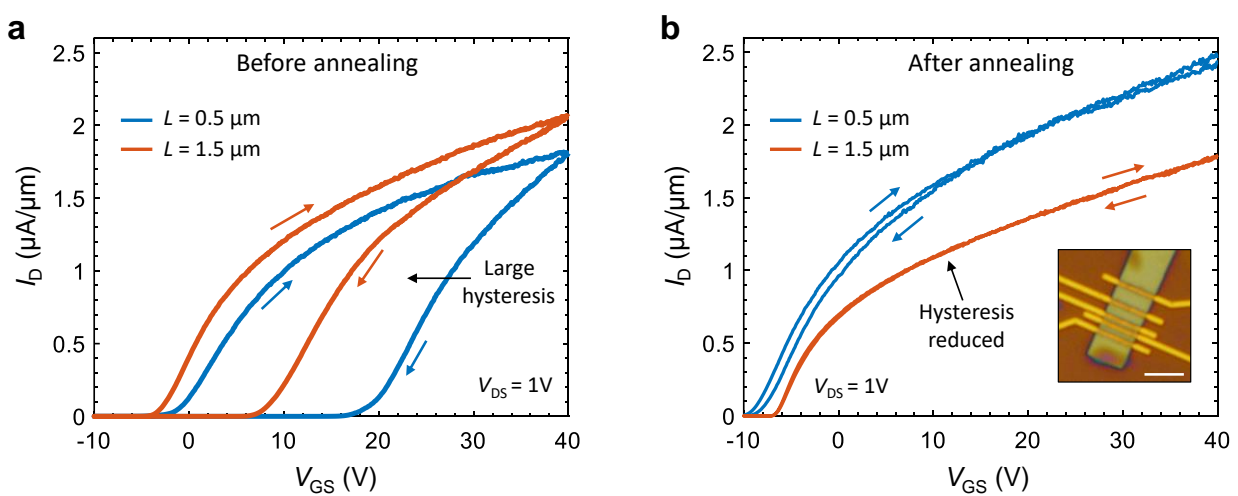

Figure S5. Hysteresis reduction by annealing. $I_{\mathrm{D}}-V_{\mathrm{GS}}$ transfer characteristics of $1 \mathrm{~L}-\mathrm{MoS}_{2}$ devices with Ti-Au (10-60 nm) edge contacts, (a) as-fabricated and (b) after annealing in $\mathrm{Ar}+\mathrm{H}_{2}$ at $200^{\circ} \mathrm{C}$ for $3 \mathrm{~h}$. The as-fabricated devices exhibit a large hysteresis between the forward and reverse sweeps, which nearly vanishes upon annealing, accompanied by a shift in the threshold voltages to lower values. The gate voltage sweep directions have been indicated by arrows. Inset: Optical image of the measured devices. In this sample, the $\mathrm{SiO}_{2}$ thickness was $285 \mathrm{~nm}$ and no in-situ $\mathrm{Ar}^{+}$sputtering was performed (which explains the lower $I_{\mathrm{D}}$ compared to Fig. S4). Scale bar: $4 \mu \mathrm{m}$. 


\section{S3. Data from additional devices}

Here we show I-V data from devices in which edge contacts were etched with $\mathrm{SF}_{6}+$ Ar. The $\mathrm{MoS}_{2}$ flake shown in Fig. S6a was exfoliated on PDMS and transferred to hBN (Fig. S6b-c). After encapsulating the $\mathrm{MoS}_{2}$ with another hBN and patterning the resulting stack into two rectangular segments, edge contacts were fabricated on each (Fig. S6d). The $I_{\mathrm{D}}-V_{\mathrm{DS}}$ characteristics of one such device are plotted in Fig. S6e and display a nearly linear behavior without any sign of saturation, at least until 3 V. In Fig. S6f, the $I_{\mathrm{D}}-V_{\mathrm{GS}}$ curves of two representative devices are shown and exhibit very similar characteristics, indicating a low-variability in contacts etched with $\mathrm{SF}_{6}+\mathrm{Ar}$, as discussed in Section $\mathrm{S} 1$ above. All $I_{\mathrm{D}}-V_{\mathrm{GS}}$ curves in Fig. S6f have been fitted with the model used in the main text. The excellent quality of the fits further corroborates the validity of the model. Log-scale $I_{\mathrm{D}}-V_{\mathrm{GS}}$ plots are shown in Fig. S6g, revealing a steep subthreshold slope and high on/off ratio, similar to Fig. 2c of the main text. These measurements, together with those in the main text, demonstrate that with our fabrication procedure, edge contacts can be made reproducibly.

a

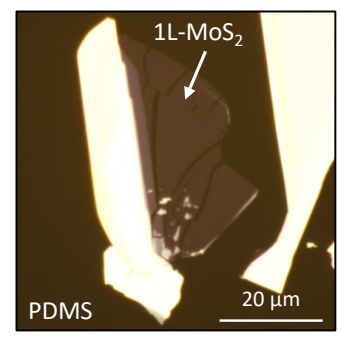

b

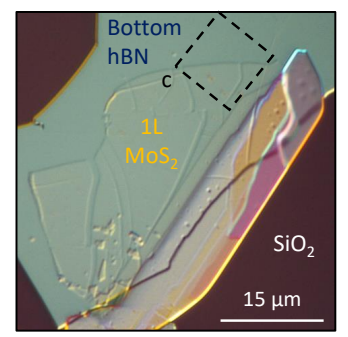

C

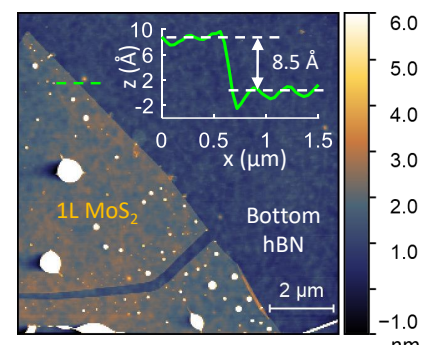

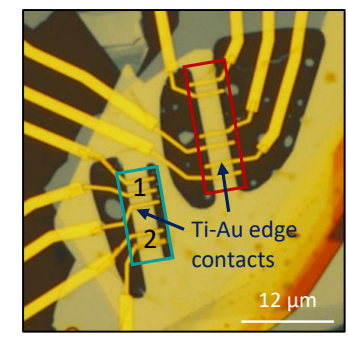
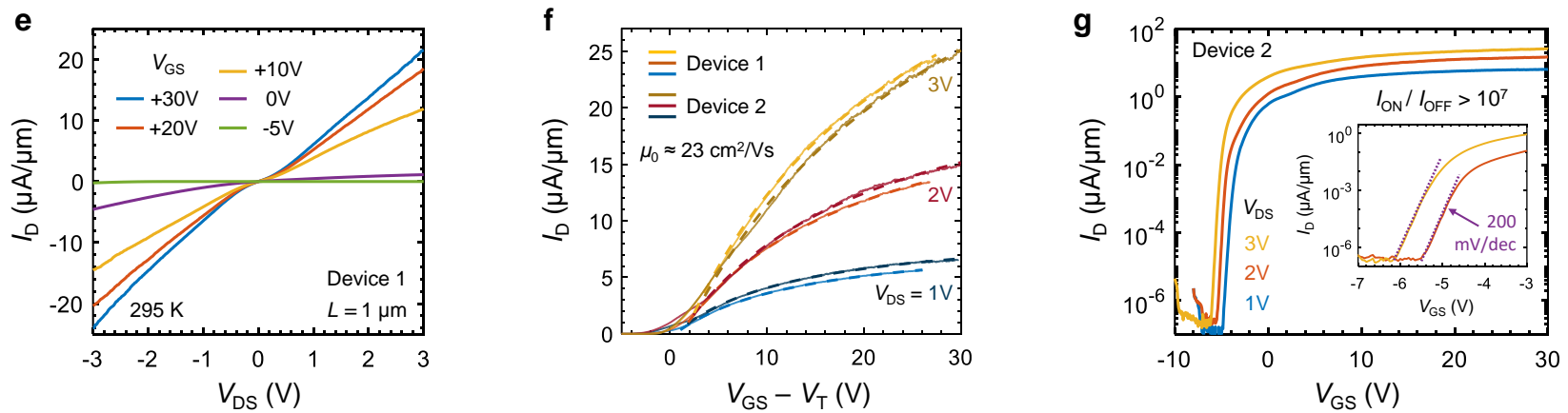

Figure S6. $\mathbf{S F}_{6}+$ Ar etched devices. (a) Optical image of a $1 \mathrm{~L}-\mathrm{MoS}_{2}$ flake exfoliated on $\mathrm{UV}_{-} \mathrm{O}_{3}$-cleaned PDMS. (b) Differential interference contrast (DIC) image of the same flake after having been transferred to hBN ( $25 \mathrm{~nm}$ thick) and vacuum annealed. (c) AFM topography map of the $10 \mu \mathrm{m} \times 10 \mu \mathrm{m}$ region outlined in $\mathbf{b}$, displaying a pristine $\mathrm{MoS}_{2}$ surface with few interfacial bubbles (bright spots). Inset: Cross-section profile along the green dashed line, revealing a thickness close to monolayer. (d) Optical image of six devices built using the MoS $\mathrm{S}_{2}$ flake in $\mathbf{b}$ after hBN encapsulation. In all devices, $L=1 \mu \mathrm{m}, W=3 \mu \mathrm{m}$ and the contact length $L_{\mathrm{c}}=0.5 \mu \mathrm{m}$. The edge contacts outlined in blue were etched by RIE with $\mathrm{SF}_{6}+\mathrm{Ar}$ while those in red with $\mathrm{CHF}_{3}+\mathrm{O}_{2}$. (e) $I_{\mathrm{D}}-V_{\mathrm{DS}}$ characteristics of the device labeled as 1 in $\mathbf{d}$, exhibiting almost symmetric and linear (for $V_{\mathrm{DS}}>0.5 \mathrm{~V}$ ) transport behavior up to $3 \mathrm{~V}$. (f) $I_{\mathrm{D}}-V_{\mathrm{GS}}$ characteristics of identical devices 1 and 2 showing very similar current densities. The dashed curves are fits obtained using the model in Eq. 4 (main text). The respective threshold voltages $V_{\mathrm{T}}$, extracted from the fits, were subtracted from $V_{\mathrm{GS}}$ in all plots, for a better comparison. (g) $I_{\mathrm{D}}-V_{\mathrm{GS}}$ characteristics of device 2 (same as in $\mathbf{f}$ ) plotted on a log-scale, displaying an on/off current ratio $>10^{7}$ and a steep subthreshold slope (inset), maintained up to three orders of magnitude. 


\section{S4. $\quad \mathrm{MoS}_{2}$ FETs with pure Au edge contacts}

In addition to $\mathrm{Ti}-\mathrm{Au}$, we also fabricated edge contacts with pure $\mathrm{Au}$ (i.e. without any $\mathrm{Ti}$ adhesion layer), since Au has been reported to result in low resistance contacts to $\mathrm{MoS}_{2}{ }^{8}$. Figure S7 shows the optical images of an hBN encapsulated 1L-MoS 2 sample with Au edge contacts, together with the I-V characteristics of one device. However, this sample was among the very few that were successfully fabricated since, in general, Au was found to often result in devices that did not conduct at all. Upon closer inspection of failed samples in an SEM, we noticed gaps between Au and hBN in several contacts, as shown in Fig. S8. By comparing the morphology of Au before and after annealing, we observed that during annealing at $300{ }^{\circ} \mathrm{C}$, Au tends to reflow and lose contact with hBN due to poor adhesion. This occurred despite Au deposition at an angle so as to completely cover the hBN sidewalls. Therefore, we infer that pure Au in combination with high-temperature annealing, is not suitable for edge contacts.

a

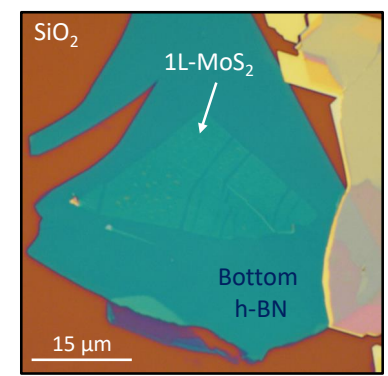

C

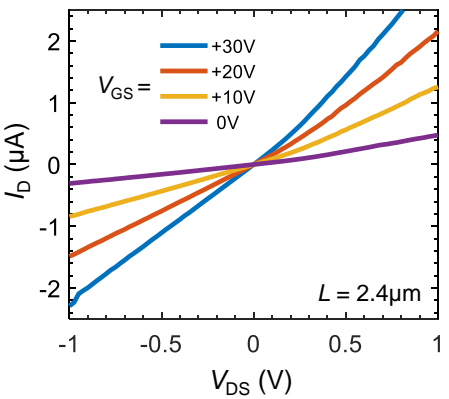

b

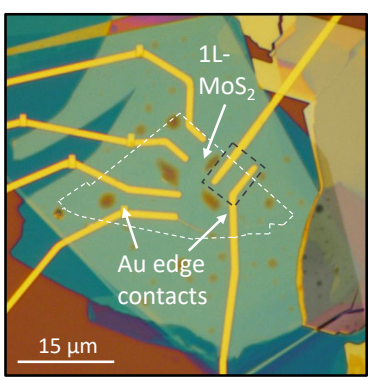

d

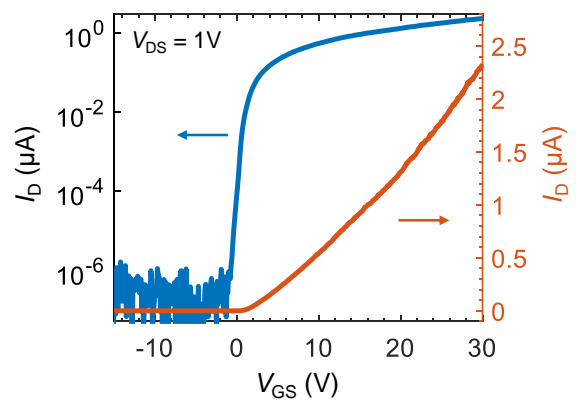

Figure S7. Pure Au edge contacts. (a) Optical image of a $1 \mathrm{~L} \mathrm{MoS}_{2}-\mathrm{hBN}$ heterostructure on a $\mathrm{Si} / \mathrm{SiO}_{2}(285 \mathrm{~nm})$ substrate. (b) Final stack after top $\mathrm{hBN}$ transfer and $\mathrm{Au}(60 \mathrm{~nm})$ edge contacts fabrication. The encapsulated $\mathrm{MoS}_{2}$ flake has been demarcated by white dashed lines. No Ar sputtering was performed for this sample. (c-d) $I_{\mathrm{D}}-V_{\mathrm{DS}}$ and $I_{\mathrm{D}}-V_{\mathrm{GS}}$ characteristics of the device outlined by the black dashed rectangle in $\mathbf{b}$.

a

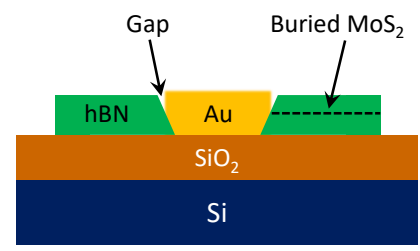

C

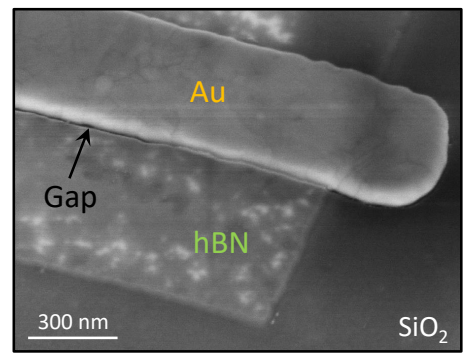

b

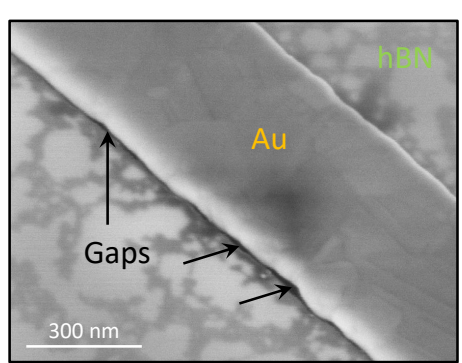

d

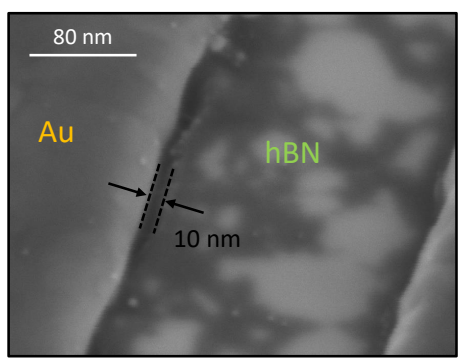

Figure S8. Poor adhesion between Au and hBN. (a) Schematic illustration of Au losing contact with $\mathrm{MoS}_{2}$ encapsulated between two hBN layers. (b-d) SEM images of actual devices revealing gaps between hBN sidewalls and Au after annealing at $300{ }^{\circ} \mathrm{C}$. Such gaps often cause devices to not conduct, making Au without an adhesion layer, highly unreliable for use in edge contacts. 


\section{S5. Edge contacts without $\mathrm{Ar}^{+}$sputtering}

In the most general case, $\mathrm{Ti}-\mathrm{MoS}_{2}$ edge contacts can be considered to be composed of $\mathrm{Ti}-\mathrm{TiO}_{\mathrm{x}}-($ air $\mathrm{molecules})-\mathrm{MoO}_{\mathrm{x}}-\mathrm{MoS}_{2}$ junctions. For improving the contact performance, it is important to determine the role of each interfacial species. As discussed in the main text, we fabricated several devices without sputtering the $\mathrm{MoS}_{2}$ contact edges with $\mathrm{Ar}^{+}$, in order to leave air molecules and $\mathrm{MoO}_{\mathrm{x}}$ (if any) intact. The transfer characteristics of three such devices, shown in Fig. S9a, exhibit a substantially lower $I_{\mathrm{D}}$ compared to $\mathrm{Ar}^{+}$sputtered devices fabricated on the same hBN-MoS 2 -hBN stack (see Fig. 1h of the main text). Furthermore, it has been found previously by X-ray photoelectron spectroscopy (XPS) ${ }^{9}$ that Ti can oxidize to form $\mathrm{TiO}_{\mathrm{x}}$ when deposited under moderately high vacuum conditions $\left(\sim 1 \times 10^{-6} \mathrm{mbar}\right)$. To inhibit $\mathrm{TiO}_{\mathrm{x}}$ formation and study its effect, Ti-Au was deposited at $\sim 1 \times 10^{-8}$ mbar on another sample (Fig. S9b), again without $\mathrm{Ar}^{+}$sputtering. Before metal deposition on the contacts, Ti (80 $\mathrm{nm}$ ) was evaporated into the chamber while keeping the sample surface covered by a shutter, to capture residual $\mathrm{O}_{2}$ molecules and lower the chamber pressure. However, as seen in Fig. S9c, $I_{\mathrm{D}}$ still remains $<1 \mu \mathrm{A} / \mu \mathrm{m}$ at $V_{\mathrm{DS}}=1 \mathrm{~V}$, irrespective of channel length $L$, revealing an $R_{\mathrm{C}}$ dominated transport in all devices. Notably, one device displayed random conductance fluctuations $(L=1 \mu \mathrm{m})$, implying an unstable contact while two others didn't conduct at all ( $L=0.5,1.5 \mu \mathrm{m})$. These results underline the importance of $\mathrm{Ar}^{+}$sputtering in realizing good edge contacts and without it, the deposition pressure (i.e. the absence or presence of $\mathrm{TiO}_{\mathrm{x}}$ ), does not make a significant difference.

a

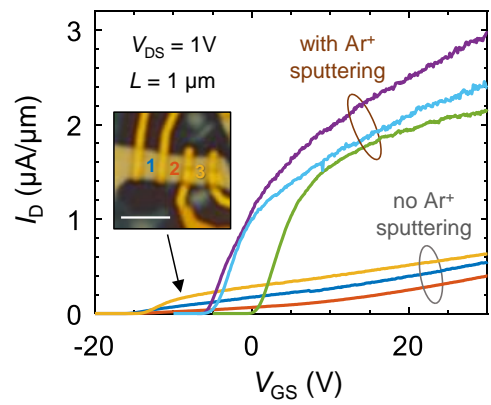

b

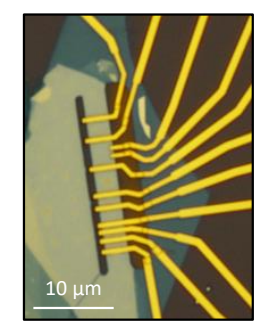

C

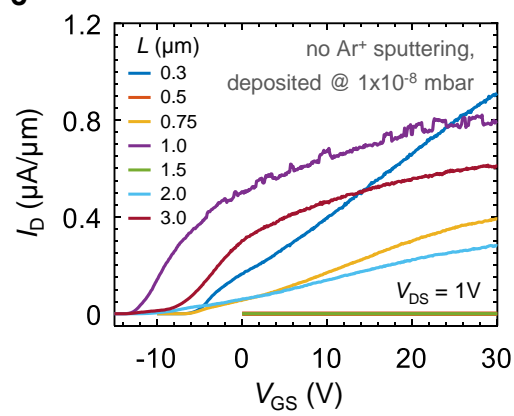

Figure S9. Devices without $\mathrm{Ar}^{+}$sputtering. (a) $I_{\mathrm{D}}-V_{\mathrm{GS}}$ characteristics of three control devices with identical dimensions (inset), also fabricated on the $1 \mathrm{~L}-\mathrm{MoS}_{2}$ flake presented in Figs. 1-2 of the main text and in Fig. S4, but without performing in-situ $\mathrm{Ar}^{+}$sputtering before metal deposition

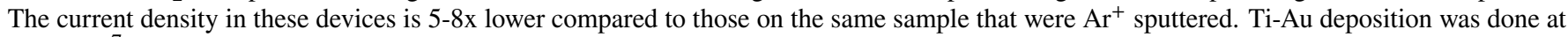
$\sim 1 \times 10^{-7}$ mbar. Inset scale bar: $3 \mu \mathrm{m}$. (b) Optical image of another set of control devices in which, $\mathrm{Ar}^{+}$sputtering was again not performed and additionally, Ti-Au was deposited at a base pressure of $\sim 1 \times 10^{-8}$ mbar to prevent $\mathrm{TiO}_{\mathrm{x}}$ deposition. The channel lengths $(L)$ range from $0.25-3 \mu \mathrm{m}$. (c) $I_{\mathrm{D}}-V_{\mathrm{GS}}$ characteristics of the devices shown in b. Despite the higher vacuum, $I_{\mathrm{D}}$ in this case too is similar to a and much lower than that obtained after $\mathrm{Ar}^{+}$sputtering. Moreover, no correlation with $L$ is discernible, indicating a high $R_{\mathrm{C}}$ in every device. All $I_{\mathrm{D}}-V_{\mathrm{GS}}$ plots shown in a and $\mathbf{c}$ were recorded after annealing in $\mathrm{Ar}+\mathrm{H}_{2}$ at $200^{\circ} \mathrm{C}$ for $3 \mathrm{~h}$. The characteristics of un-sputtered devices did not improve considerably upon further annealing at higher temperatures. 


\section{S6. Quantum transport simulations}

To gain further insight into carrier transport through edge contacts, we performed ballistic ab initio quantum transport simulations based on density functional theory (DFT). The non-equilibrium Green's function (NEGF) formalism was used for this purpose, which involves solving the following equation,

$$
\left[E-H_{\mathrm{MLWF}}\left(k_{z}\right)-\Sigma^{\mathrm{RB}}\left(E, k_{z}\right)\right] \cdot G^{\mathrm{R}}\left(E, k_{z}\right)=I
$$

for the momentum $k_{z}$ and energy $E$ dependent retarded Green's function $G^{\mathrm{R}}\left(E, k_{z}\right)$ and boundary self-energy $\Sigma^{\mathrm{RB}}\left(E, k_{z}\right)$. The Hamiltonian matrix $H_{\mathrm{MLWF}}\left(k_{z}\right)$ was expressed in a maximally localized Wannier function (MLWF) basis. It was constructed according to the upscaling technique described in Ref. 10 for the Ti-MoS 2 edge contact structure depicted schematically in Fig. S10a. The size of $H_{\mathrm{MLWF}}\left(k_{z}\right)$ is equal to $N_{\mathrm{A}} \times N_{\text {orb }}$, where $N_{\mathrm{A}}$ is the number of atoms in the simulation domain and $N_{\text {orb }}$ is the average number of MLWF per atom. Once Eq. 1 was solved for all energies and momenta of interest, the density-of-states $\operatorname{DOS}\left(E, k_{z}\right)=\operatorname{diag}\left(G^{\mathrm{R}}\left(E, k_{z}\right)-G^{\mathrm{A}}\left(E, k_{z}\right)\right)$ and the transmission function $\mathcal{T}\left(E, k_{z}\right)=\operatorname{tr}\left(G_{1 N}^{\mathrm{R}}\left(E, k_{z}\right) \cdot \Gamma_{N N} \cdot G_{N 1}^{\mathrm{A}}\left(E, k_{z}\right) \cdot \Gamma_{11}\right) \operatorname{could}$ be computed, from which the charge density and electronic current were derived. Here, $G^{\mathrm{A}}\left(E, k_{z}\right)$ is the advanced Green's function and $\Gamma_{11 / N N}$ is the broadening function corresponding to the first (index 1) and last (index $N$ ) unit cell of the contact geometry.

The resulting spatially- and energetically-resolved $\operatorname{DOS}(E)=\sum_{k_{z}} \operatorname{DOS}\left(E, k_{z}\right)$ is displayed in Fig. S10b. From this plot, the Ti-MoS $\mathrm{S}_{2}$ band alignment and the resulting Schottky barrier height $\left(\phi_{\mathrm{SB}}\right)$ can be identified. The energy-dependent electron transmission function $\mathcal{T}(E)=\sum_{k_{z}} \mathcal{T}\left(E, k_{z}\right)$ is plotted in Fig. S10c. The total injected current density was then determined via the Landauer-Büttiker formalism by multiplying $\mathcal{T}\left(E, k_{z}\right)$ with the difference between the Fermi-Dirac distribution functions $f(E, T)$ (i.e. the occupation probability at a given temperature) of the right and left contacts and integrating the result over energy and momentum. Figure S10d shows the simulated current density as a function of temperature. Since in real devices the contact metal Fermi level is often strongly pinned close to the $\mathrm{MoS}_{2}$ conduction band minimum ${ }^{11,12}$ and because DFT, in general, does not accurately estimate the band offset between two materials, the current was calculated for several values of $\phi_{\mathrm{SB}}$. This was done by artificially raising the metal Fermi level indicated in Fig. S10b. As evident from Fig. S10d, the injected current density remains largely independent of temperature, regardless of the barrier height, which corroborates the measured $I_{\mathrm{D}}-V_{\mathrm{GS}}$ characteristics in Fig. 4a of the main text.

The relative temperature insensitivity of the current can be traced back to the underlying carrier injection mechanism and the shape of the transmission function $\mathcal{T}(E)$. In case of $\mathrm{Ti}-\mathrm{MoS}_{2}$ edge contacts, the largest contribution to the current does not come from thermionic processes but instead from quantum mechanical tunneling through the Schottky barrier, which does not heavily depend on temperature. Furthermore, the magnitude of the transmission function from Ti to $\mathrm{MoS}_{2}$ does not vary much around the Fermi energy such that the current $I_{\mathrm{D}}$ for large $V_{\mathrm{DS}}$ can be approximated as, $I_{\mathrm{D}} \approx \frac{e}{\hbar} \int_{E_{\min }}^{\infty} C \cdot f\left(E, E_{\mathrm{F}}^{\mathrm{M}}, T\right) \mathrm{d} E$, where $E_{\text {min }}$ corresponds to the conduction band minimum of $\mathrm{MoS}_{2}$ far from the interface region, $E_{\mathrm{F}}^{\mathrm{M}}$ is the Fermi level of the metal contact and $C$ is a constant. It can be shown that such an expression exhibits little to no dependence on the temperature $T$.
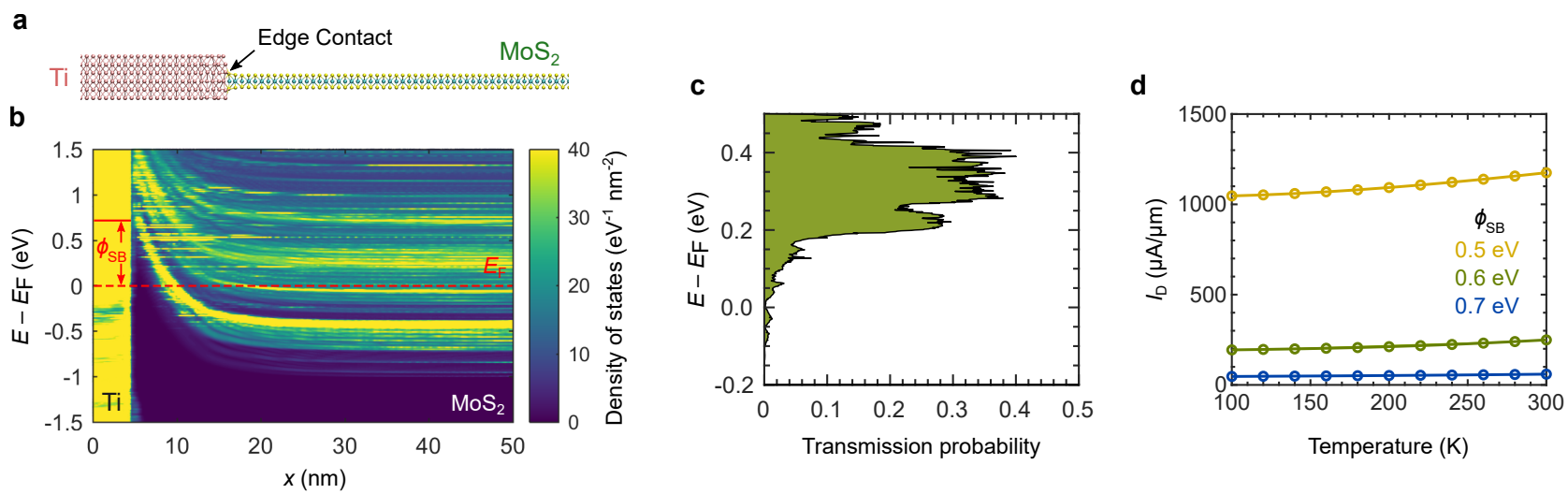

Figure S10. Quantum transport simulations of edge contacts. (a) Schematic cross-section and (b) computed band-diagram of a Ti - monolayer MoS 2 edge contact structure. The color scale in (b) represents the DOS per unit area plotted on a linear scale, the horizontal red dashed line denotes the Fermi level $E_{\mathrm{F}}$ and $\phi_{\mathrm{SB}}$ indicates the Schottky barrier height. The effect of an applied gate bias was incorporated by shifting the MoS 2 conduction band minima to $1 \mathrm{eV}$ below $E_{\mathrm{F}}$. (c) Energy dependent electron transmission probability $\mathcal{T}(E)$ for the DOS distribution shown in b. (d) Total current density as a function of temperature, calculated for three different $\phi_{\mathrm{SB}}$ values. We find that the injected current density depends only weakly on temperature for $\mathrm{T}<300 \mathrm{~K}$ since the transmission function in $\mathbf{c}$ does not change appreciably around the Fermi level. 
*acjain@ethz.ch

$\dagger$ lnovotny@ethz.ch

${ }^{1}$ Glasmästar, K., Gold, J., Andersson, A.-S., Sutherland, D. S. \& Kasemo, B. Silicone transfer during microcontact printing. Langmuir 19, 5475-5483 (2003).

2 Jain, A. et al. Minimizing residues and strain in 2D materials transferred from PDMS. Nanotechnology 29, 265203 (2018).

3 Ouyang, M., Yuan, C., Muisener, R. J., Boulares, A. \& Koberstein, J. T. Conversion of some siloxane polymers to silicon oxide by UV/ozone photochemical processes. Chem. Mater. 12, 1591-1596 (2000).

4 Wang, L. et al. One-dimensional electrical contact to a two-dimensional material. Science 342, 614-617 (2013).

5 Autore, M. et al. Boron nitride nanoresonators for phonon-enhanced molecular vibrational spectroscopy at the strong coupling limit. Light Sci. Appl. 7, 17172 (2018).

6 Wu, D. et al. Uncovering edge states and electrical inhomogeneity in $\mathrm{MoS}_{2}$ field-effect transistors. Proc. Natl. Acad. Sci. USA 113, $8583-8588$ (2016).

7 K. C., S., Longo, R. C., Addou, R., Wallace, R. M. \& Cho, K. Electronic properties of $\mathrm{MoS}_{2} / \mathrm{MoO}_{\mathrm{x}}$ interfaces: Implications in tunnel field effect transistors and hole contacts. Sci. Rep. 6, 33562 (2016).

8 Smithe, K. K., English, C. D., Suryavanshi, S. V. \& Pop, E. Intrinsic electrical transport and performance projections of synthetic monolayer $\mathrm{MoS}_{2}$ devices. 2D Materials 4, 1-8 (2017).

9 McDonnell, S., Smyth, C., Hinkle, C. L. \& Wallace, R. M. $\mathrm{MoS}_{2}$-titanium contact interface reactions. ACS Appl. Mater. Interfaces 8 , 8289-8294 (2016).

10 Szabó, A., Jain, A., Parzefall, M., Novotny, L. \& Luisier, M. Electron transport through metal/MoS 2 interfaces: Edge- or area-dependent process? Nano Lett. 19, 3641-3647 (2019).

11 Das, S., Chen, H.-Y., Penumatcha, A. V. \& Appenzeller, J. High performance multilayer $\mathrm{MoS}_{2}$ transistors with scandium contacts. Nano Lett. 13, 100-105 (2013).

12 Kim, C. et al. Fermi level pinning at electrical metal contacts of monolayer molybdenum dichalcogenides. ACS Nano 11, 1588-1596 (2017). 